\section{OPEN ACCESS}

Edited by:

Isabel Merida,

Consejo Superior de Investigaciones Cientificas (CSIC), Spain

Reviewed by: Jurgen Muller,

University of Bradford, United Kingdom

Marco A. Briones-Orta

Imperial College London, United Kingdom

*Correspondence:

Eleni Anastasiadou eleni.anastasiadou@uniroma1.it Lisa M. Minter

Iminter@vasci.umass.edu Maria Pia Fell mariapia.felli@uniroma1.it

${ }^{\dagger}$ These authors have contributed equally to this work

Specialty section:

This article was submitted to

Signaling,

a section of the journal

Frontiers in Cell and Developmental

Biology

Received: 06 April 2021

Accepted: 20 July 2021

Published: 05 August 2021

Citation:

Trivedi P, Patel SK, Bellavia D, Messina E, Palermo R, Ceccarelli S,

Marchese $C$, Anastasiadou E, Minter LM and Felli MP (2021) When Viruses Cross Developmental

Pathways.

Front. Cell Dev. Biol. 9:691644. doi: 10.3389/fcell.2021.691644

\title{
When Viruses Cross Developmental Pathways
}

\begin{abstract}
Pankaj Trivedi', Sandesh Kumar Patel ${ }^{2}$, Diana Bellavia ${ }^{2}$, Elena Messina ${ }^{1}$, Rocco Palermo ${ }^{2}$, Simona Ceccarelli ${ }^{1}$, Cinzia Marchese ${ }^{1}$, Eleni Anastasiadou ${ }^{1 * t}$, Lisa M. Minter ${ }^{3 * t}$ and Maria Pia Felli ${ }^{* t}$
\end{abstract}

'Department of Experimental Medicine, Sapienza University of Rome, Rome, Italy, ${ }^{2}$ Department of Molecular Medicine, Sapienza University of Rome, Rome, Italy, ${ }^{3}$ Department of Veterinary and Animal Sciences, University of Massachusetts Amherst, Amherst, MA, United States

Aberrant regulation of developmental pathways plays a key role in tumorigenesis. Tumor cells differ from normal cells in their sustained proliferation, replicative immortality, resistance to cell death and growth inhibition, angiogenesis, and metastatic behavior. Often they acquire these features as a consequence of dysregulated Hedgehog, Notch, or WNT signaling pathways. Human tumor viruses affect the cancer cell hallmarks by encoding oncogenic proteins, and/or by modifying the microenvironment, as well as by conveying genomic instability to accelerate cancer development. In addition, viral immune evasion mechanisms may compromise developmental pathways to accelerate tumor growth. Viruses achieve this by influencing both coding and non-coding gene regulatory pathways. Elucidating how oncogenic viruses intersect with and modulate developmental pathways is crucial to understanding viral tumorigenesis. Many currently available antiviral therapies target viral lytic cycle replication but with low efficacy and severe side effects. A greater understanding of the cross-signaling between oncogenic viruses and developmental pathways will improve the efficacy of next-generation inhibitors and pave the way to more targeted antiviral therapies.

Keywords: Hedgehog, Notch, WNT, oncogenic viruses, immune evasion, microRNA, targeted therapies

\section{INTRODUCTION}

About $15 \%$ of all human tumors have infectious etiology and yet only a handful of viruses are known to promote tumor development (White et al., 2014). Clearly, tumor development is neither the aim of the virus nor it is required for virus transmission. The evolutionary mechanisms of viral replication, establishment of latency and immune evasion are often the underlying causes that make the infected cell emancipate from normal proliferation dictated by homeostasis. Discovering oncogenic virus-targeted genes and developmental signaling pathways is imperative to understanding viral tumorigenesis. Viruses hijack different cellular programs with the aim to survive and replicate within its host. By interacting with host proteins, they perturb and interfere with host signaling pathways to modify critical cellular functions. Integration of the viral genome into the host DNA may be a critical factor in carcinogenesis, particularly for some Human Papilloma virus (HPV) serotypes (Oyervides-Muñoz et al., 2018). Herpesviruses on the other hand, mainly remain as extra-chromosomal DNA in the host cell nucleus. In both cases, viruses alter expression and function of genes primarily associated with cell proliferation, differentiation, and survival, and induce chromosomal instability chromosomal instability.

In recent years, computational reconstruction of proteome-wide protein-protein interaction (PPI) networks between viruses and developmental pathways have enhanced our understanding 
of virus-induced carcinogenesis. Mei and Zhang studied PPI networks to highlight important relationships between Epstein-Barr virus (EBV) proteins and developmental pathways, including Hedgehog $(\mathrm{HH})$ and Notch signaling (Mei and Zhang, 2016). By exploiting components of the $\mathrm{HH}$ pathway, viruses promote tumor growth, survival, and stemness-associated programs in order to transform infected cells. Therefore, $\mathrm{HH}$ targeted therapies could represent a promising strategy to combat virus-induced tumors. Another study demonstrated that Hepatitis B Virus (HBV) DNA integration preferentially targets cancer related pathways such as MAPK, extracellular matrix (ECM)-receptor interactions, and the $\mathrm{HH}$ signaling pathways (Yang et al., 2018).

The Notch pathway is evolutionarily conserved and participates in a plethora of physiological intercellular and intracellular signaling processes during differentiation and development of an organism. Evidence shows that virally perturbed Notch signaling may lead to cancer (Meisel et al., 2020). Oncogenic viruses also exploit Notch pathway to escape immune recognition and facilitate their own survival in the host to enhance infectivity and transmission. Remarkably, several viral oncoproteins, such as Epstein-Barr nuclear antigen 2 (EBNA2), Hepatitis $\mathrm{Bx}(\mathrm{HBx})$, latency-associated nuclear antigen (LANA) of Kaposi's sarcoma-associated herpes virus (KSHV, also known as HHV-8) and others, interact with several members of the Notch pathway (Hayward, 2004).

The WNT signaling developmental program is also frequently targeted by oncogenic viruses to transform the target cells. HBV, Hepatitis C virus (HCV), EBV and Human T Lymphocyte Virus-1 (HTLV-1) co-opt or modulate components of the WNT pathway to effectively subvert normal cellular processes including cell proliferation, differentiation, and survival.

Thus, as our understanding of the mechanisms that regulate oncogenic transformation grows, the extent and complexity of cellular processes targeted by oncoviruses is better appreciated. The recognition that multiple developmental pathways are frequently targeted, either individually or collectively, may represent unexplored opportunities for developing unique or synergistic therapeutic strategies to treat or prevent viral tumorigenesis.

In this review, we present an overview of the three developmental pathways, namely, $\mathrm{HH}$, Notch, and WNT and how some oncogenic viruses interact with them. We will review immune system interactions with these viruses, and how they regulate these pathways through viral miRNAs to survive and contribute to carcinogenesis and tumor progression. We will also provide perspectives for the development of therapies that target important and common regulators of these three developmental pathways.

\section{HH SIGNALING IN VIRAL ONCOGENESIS}

\section{Overview of HH Signaling}

Tissue patterning, cell differentiation and proliferation require $\mathrm{HH}$ signaling but aberrant $\mathrm{HH}$ signaling is an important cause of cancer. In humans, the $\mathrm{HH}$ pathway is activated by three ligands: Sonic Hedgehog (SHH), Indian Hedgehog ( $\mathrm{IHH})$, and Desert Hedgehog (DHH) (Qi and Li, 2020). These ligands share a high degree of sequence and functional homology and act to initiate this paracrine signaling cascade. The two Patched genes, PTCH1 and PTCH2 in humans, code for the primary receptors of $\mathrm{HH}$ ligands, consisting of 12 transmembrane helices (TMs), three extracellular domains (ECDs), and one C-terminal domain (CTD). The two receptors share a conserved TM domain and two of three ECDs. In contrast, PTCH2 lacks the CTD domain. After binding $\mathrm{HH}, \mathrm{PTCH} 1$ is inhibited and forms oligomers, which are further moved out of the cilia and degraded in the endosome (Tukachinsky et al., 2016). In so doing, Smoothened (SMO) is no longer inhibited and this Frizzle-class $\mathrm{G}$ protein-coupled receptor (GPCR) can relocate to the cilia, a small organelle extending from the plasma membrane, which provides a localized hub in which transmembrane receptors can concentrate (Figure 1). SMO signals through at least two effector routes. The first is a $\mathrm{G}$ protein-independent, canonical pathway that signals to three members of the glioma-associated (GLI) oncogene family, with the aim to upregulate target genes (Figure 1). One of them, Hip (Figure 1), a $\mathrm{HH}$ interacting protein attenuates ligand diffusion (Arensdorf et al., 2016). In vertebrates, suppressor of Fused (SUFU) represses GLI transcription factor activation and the active SMO releases this inhibition. GLI1 functions as a feed-forward activator to sustain or amplify target gene expression (Pandit and Ogden, 2017). GLI2 and GLI3 are bifunctional and can be processed to act either as transcriptional activators or repressors (Crompton et al., 2007). Ciliary SMO signaling halts GLI processing, further stabilizing GLI2 and GLI3 as transcriptional activators of $\mathrm{SHH}$ target genes, such as GLI1. The second route, referred to as the non-canonical SMO signaling pathway, triggers transcription-independent responses that are fundamental to establishing and maintaining distinct cell behaviors during development (Pandit and Ogden, 2017). Involvement of the non-canonical SMO signaling in viral carcinogenesis is yet to be fully explored (Palle et al., 2015).

\section{HH Signaling Targeted by Oncogenic Viruses Promotes Tumor Development}

In addition to its indispensable role in developmental processes, more than $25 \%$ of all cancers require autocrine or paracrine $\mathrm{HH}$ signaling as a fundamental supporter of tumor cell growth and survival (Figure 1) (Lum and Beachy, 2004; Iriana et al., 2021). In EBV-positive nasopharyngeal carcinomas (NPC) and EBV-infected epithelial cell lines, the virus can activate the $\mathrm{HH}$ signaling pathway through autocrine induction of $\mathrm{SHH}$ (Port et al., 2013). This is corroborated by the expression of $\mathrm{HH}$ pathway effectors (GLI1 and GLI2) and $\mathrm{HH}$ target genes, such as PTCH1, FOXM1, and WNT5A, a highly evolutionarily conserved non-canonical WNT ligand. Altered $\mathrm{HH}$ signaling is common in NPC and specifically a reduced expression of SUFU has been detected in a large number of NPC specimens (Port et al., 2013). An $\mathrm{HH}$ autocrine signaling loop has also been associated with HPV infection, a 'primary hit' in cervical cancer (CC) development. Tumor cells express $\mathrm{HH}$ pathway components, and $\mathrm{HH}$ signaling promotes proliferation, survival, 


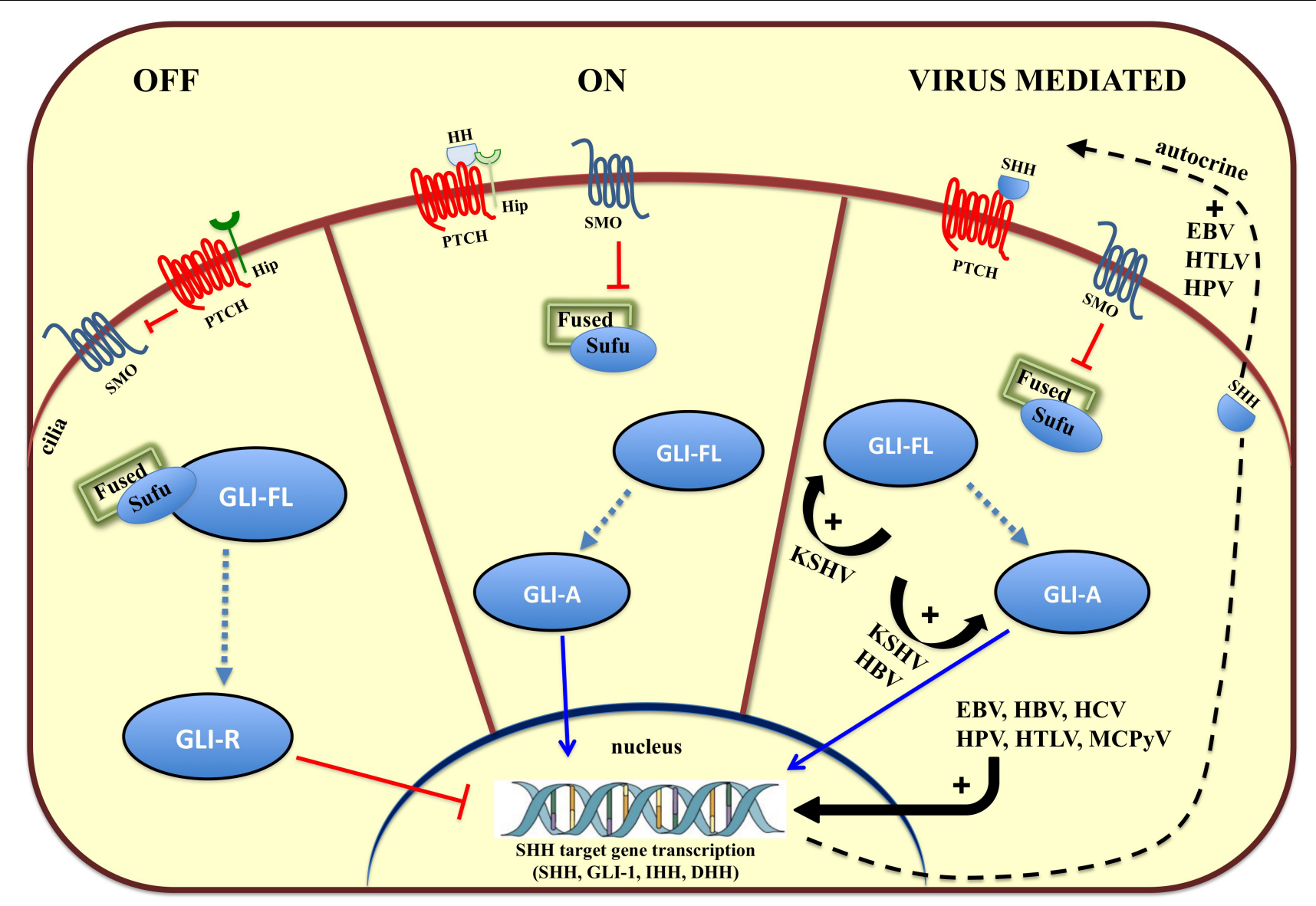

FIGURE 1 | Overview of the Hedgehog $(\mathrm{HH})$ signaling pathway and modulation by oncoviruses. On the left, in the absence of $\mathrm{HH}$ ligands $(\mathrm{SHH}, \mathrm{IH} H$, and $\mathrm{DH} H)$, the $\mathrm{HH}$ receptor Patched (PTCH) keeps the pathway off (OFF) by inhibiting Smoothened (SMO), and keeping the glioma-associated oncogene (GLI) transcription factors in an inactive form (GLI-R). In the middle, binding of $\mathrm{HH}$ ligand to $\mathrm{HH}$ receptor, $\mathrm{PTCH}$, turns the pathway on (ON), and inhibits its activity, relieving the repression of SMO, which converts full-length GLI (GLI-FL) into a transcriptional activator (GLI-A). In vertebrates, cilia are required for production of GLI-repressor (GLI-R) and/or GLI-activator (GLI-A). On the left, viruses hyperactivate HH signaling with multiple mechanisms (VIRUS MEDIATED). EBV, Epstein Barr Virus; HBV, Hepatitis B Virus; HCV, Hepatitis C Virus; HPV, Human Papilloma Virus, KSHV, Kaposi's Sarcoma-associated Herpes Virus; MCPyV, Merkel Cell Polyoma Virus.

and migration of CC cells. These pro-survival and protective roles are prevented by a small molecule inhibitor that blocks binding of Gli to DNA, GANT-61, which induces caspase 3 cleavage indicating an increased apoptosis in human CC cell lines (Samarzija and Beard, 2012).

Viruses can also epigenetically affect factors involved in cellular $\mathrm{HH}$ activation, which implies that they may participate directly in configuring chromatin architecture. This is the case of a malignant $\mathrm{T}$ cell disorder caused by infection with the human retrovirus, $H T L V-1$. Indeed, in adult $\mathrm{T}$ cell leukemia (ATL), the HTLV-1 TAX transcription factor epigenetically upregulates Ellis Van Creveld (EVC) family members, EVC1 and EVC2, both of which have been associated with the cellular $\mathrm{HH}$ activity and thus provides the pro-survival attributes of ATL cells (Takahashi et al., 2014). Additionally, TAX can also induce $\mathrm{SHH}$ transcription in an NF- $\kappa \mathrm{B}$-dependent manner to sustain $\mathrm{HH}$ autocrine stimulation in malignant cells (Figure 1). Besides directly activating $\mathrm{HH}$ signaling, oncogenic viruses may exploit the connection between the transcription factor,
ZIC2, and the microRNA (miRNA), miR-129-5p, as recently reported in lymphangiogenesis and lymph node metastasis during NPC progression (Yu et al., 2020). Importantly, it has been demonstrated that EBV, a virus closely associated with NPC, may down-regulate miR-129 expression (Forte and Luftig, 2011). This leads to ZIC2 activation, a zinc-finger transcription factor that upregulates $\mathrm{HH}$ related signaling molecules, SMO, GLI1, and SHH. Additionally, Yu et al. (2020) demonstrate that miR-129-5p overexpression silences ZIC2 and decreases NPC cell proliferation, migration, and invasiveness, suggesting that miR-129-5p may serve as a novel therapeutic tool for NPC.

Infection with oncogenic viruses may be silent for years and $\mathrm{HH}$ pathway reactivation later in life has been associated with tumor development (Kuromi et al., 2017). Merkel cell polyomavirus (MCPyV) is detected in approximately $80 \%$ of Merkel cell carcinoma (MCC) (Harms et al., 2018). It is an aggressive neuroendocrine skin cancer that mainly affects the elderly. In this case, higher expression of SHH and GLI1 were significantly associated with a favorable prognosis and represent 
useful markers of MCC (Kuromi et al., 2017). Intriguingly, the interaction between $\mathrm{MCPyV}$ and developmental pathways has remained hitherto unexplored.

In EBV-associated tumors, the virus persists in one of the three distinct latent phases, each characterized by expression of a set of viral genes. In NPC, EBV latent gene expression is restricted to EBNA1, EBV-encoded RNA1/2 (EBER1/2), BARF1, the BamHIA transcripts (BARTs), as well as variable expression of genes encoding oncogenic membrane proteins LMP1 and LMP2. In these tumors, EBNA1, LMP1 and LMP2 all stimulate $\mathrm{HH}$ signaling, but only the latter two are capable of reprogramming cells toward stemness. This would suggest that each viral protein can trigger different molecular pathways involved either in tumor cell growth and survival, or in stemness-associated programs. Just like normal stem cells, cancer cells have the ability to selfrenew, differentiate in different cell types and under specific tumor microenvironmental condition, dedifferentiate backward a primitive state. This feature provides tumor cells the ability to grow, metastasize and home to specific tissues. Given its ability to activate the WNT and Notch pathways, Port et al. (2013) suggested LMP2A may impose stem-like characteristics on EBV-infected epithelial cells by recruiting additional oncodevelopmental pathways. Indeed, the $\mathrm{HH}$ pathway, together with WNT and Notch signaling, maintain a population of normal stem cells and transforms cancer stem cells by inducing stemnessassociated gene expression (Katoh, 2007). Overall, EBV and other oncogenic viruses trigger the $\mathrm{HH}$ autocrine signaling loop, a fundamental mechanism in cell survival and proliferation.

\section{HH Pathway Mediates Virus-Induced Epithelial to Mesenchymal Cell Transition (EMT)}

Co-expression of cancer stem/initiating and mesenchymal cell markers have been observed in peritumoral stromal tissues within nodules of Hepatocellular Carcinoma (HCC) from livers of patients with chronic hepatitis. Both $\mathrm{HBV}$ and $\mathrm{HCV}$ clearance rates are high in infected individuals, often depending on the age (Chou et al., 2015). Only about $5-10 \%$ infected individuals become chronic carriers. In case of HBV, integration of the viral genome plays an important role in both chronic carrier state and subsequent diseases (Chou et al., 2015). Indeed, the chronically infected individuals have higher risk of developing HCCs. Other genetic events like mutation and/or inactivation of p53 are also frequent (Feitelson et al., 1993). HBx protein of HBV directly binds p53 and sequesters it in the cytoplasm thus compromising its ability to induce apoptosis (Elmore et al., 1997).

The $\mathrm{HH}$ signaling pathway is maintained in an inactive state in a healthy adult liver due to high levels of Hip, which disrupts engagement between the $\mathrm{HH}$ ligand and the receptor (Hyun and Jung, 2016). Gene profiling studies of human liver cancers provide evidence that chronic infection by $\mathrm{HBV}$ and $\mathrm{HCV}$ significantly increases hepatic mRNA expression of $\mathrm{HH}$-ligands, SHH and $I H H$, and target genes, PTCH and GLI2, possibly during more advanced stages of liver disease (Pereira Tde et al., 2010). Indeed, Pereira Tde et al. (2010) reported that the fibrosis stage and HCC development are predicted to increase in parallel with the level of $\mathrm{HH}$ pathway activity. As the level of $\mathrm{HH}$ ligands increases, hepatic accumulation of $\mathrm{HH}$-reactive progenitors also increases, concurrent with a decreased Hip expression (Hyun and Jung, 2016). The EMT transition is characterized by epithelial cells losing their polarity, the ability of cell-cell contact and acquiring features which make them resemble mesenchymal cells. While EMT occurs physiologically during embryogenesis, this transition is also a characteristic of many neoplastic diseases. The loss of E-cadherin by epithelial cells is considered the cornerstone of EMT (Kalluri and Weinberg, 2009). As such, Pereira Tde et al. (2010) reported that an increased activity of $\mathrm{HH}$ signaling in chronic viral hepatitis correlates to the enriched subpopulation of $\mathrm{HH}$-responsive progenitors that are undergoing EMT in hepatic nodules. Cellular migration is an integral step in EMT that results in liver remodeling in chronic liver disease and promotes metastasis during cancer progression (Arzumanyan et al., 2012). This event is largely dependent on activation of $\mathrm{HH}$ signaling ( $\mathrm{SHH}$ and GLI2) by the HBV transcription factor, $\mathrm{HBx}$, in HCC pathogenesis. The mechanism by which HBx upregulates the expression of $\mathrm{HH}$ components, either through transcriptional control (Arzumanyan et al., 2012) or through post-translational stabilization and nuclear localization (Kim et al., 2011), remains to be fully elucidated. Moreover, $\mathrm{HBx}$ also promotes stemness in the liver (Arzumanyan et al., 2011). Perhaps, the discovery that $\mathrm{HBx}$ activates $\mathrm{HH}$ signaling in the pathogenesis of HCC may lead to therapies that are better targeted to prevent tumor initiation and/or that block the growth and relapse of established tumors. Interestingly, in hepatic carcinogenesis EMT and enhanced $\mathrm{HH}$ signaling activation have been suggested to promote chemoresistance and invasion of poorly differentiated hepatoma cells often negative for CD133 and EpCAM. These observations may provide a new basis for reclassifying HCC specimens and may represent promising targets in eradicating chemoresistant subpopulations in HCC (Chen et al., 2011).

\section{Developmental Pathways Are Not the Only Downstream Targets of Oncogenic Viruses}

Although the role of viral oncoproteins E6 and E7 in HPVmediated cervical carcinogenesis is well-established, still to be studied is the interaction of GLI signaling with HPV encoded oncogenes. Recently, active GLI signaling has been demonstrated in CC cells irrespective of the presence of HPV and was associated with cell viability. Inhibiting GLI signaling in HPV-positive CC cells is associated with reduced HPV E6 oncogene expression and loss of stemness (Vishnoi et al., 2016). Conversely, silencing the HPV-16 E6 oncogene reduced GLI1 transcription. This reciprocal interference suggests a cooperation between viral and $\mathrm{HH}$ proteins. Indeed, inhibiting both E6 and GLI signaling produces an additive effect on cell viability, leading to the hypothesis that they synergize to promote stemness in CC cells. Loss of p53, triggered by HPV E6, is a probable connecting link with constitutively active GLI signaling observed during persistent high risk HPV infection. Additionally, GLI-HPV E6 cooperation sustains cancer cell stemness possibly leading to 
tumor progression and chemoresistance, as observed in clinically advanced CC (Vishnoi et al., 2016).

According to Piirsoo et al. (2019) full-length GLI has a role in regulating HPV transcription and, surprisingly, it acts as a repressor. Human GLI1 has at least three alternatively spliced isoforms, including GLI $\Delta \mathrm{N}$ that lacks the conserved phosphorylation cluster and the SUFU binding motif. Shuttling of GLI1 between the cytoplasmic and nuclear compartments depends on several factors, including interaction with SUFU and Protein Kinase A-mediated phosphorylation. These authors further demonstrate that, in contrast to GLI1 $\Delta \mathrm{N}$, full-length GLI1 suppresses replication of multiple HPV genomes, including HPV-5, -11, and -18. Non-productive infections often terminate the viral life cycle and may be crucial for HPV-DNA persistence and tumorigenesis (Gaglia and Munger, 2018). According to the bioinformatic analysis, the predicted GLI1 binding site mostly overlaps with the viral E2 binding site, suggesting a direct impact on the initiation of HPV5 replication. Overall, this observation suggests there may be differing contributions of GLI1 isoforms to the HPV lifecycle and, interestingly, highlights the regulatory role of Gli1 on HPV transcription.

Viral carcinogenesis may also rely on the ability of oncogenic viruses to select a suitable genetic cell environment without directly targeting $\mathrm{HH}$ signaling. Cervical carcinogenesis is a multistep process. HPV infection is not sufficient per se, but provides a 'second hit', most likely through moderate levels of Notch1 and the cooperation of $\mathrm{HH}$ and WNT signals to transform keratinocytes (Lathion et al., 2003). In this context, $\mathrm{HH}$ signaling is not triggered directly by HPV E6 and E7 proteins but, rather, that $\mathrm{HH}$-activating mutations are selected in cells initially immortalized by HPV (Samarzija and Beard, 2012). Therefore, the dual role of $\mathrm{HH}$ signaling, serving either as a collaborator of HPV-induced carcinogenesis or as a regulator of viral oncogene expression stresses the critical role of $\mathrm{HH}$ inhibitors as a therapeutic option in CC.

\section{Virus-Induced Tumor Progression and Immune Evasion Strategies Point to GLI1 Activity}

Recently, Asha et al. (2020) reported on the unexplored function of $\mathrm{HH}$ signaling in regulating the biology of latent and lytic states of sarcoma KSHV. This virus, also known as human herpesvirus 8 (HHV8) hijacks pro-inflammatory pathways and concurrently reduces anti-inflammatory Lipoxin A4 (LXA4) secretion to maintain the virus in a latent state. In KS skin tissue, GLI1 is significantly increased and is distributed both in the cytoplasm and nucleus (Figure 1). This is in contrast to healthy tissue in which GLI1 is expressed exclusively in the nuclei. LXA4-treated KSHV-infected cells showed decreased GLI1 expression, independent of $\mathrm{SHH}$ modulation, and mainly through GLI1 destabilization, which may also decrease the angiogenic processes. In fact, GLI1 can transcriptionally upregulate vascular endothelial growth factor C (VEGF-C) expression to promote angiogenesis (Carpenter and Lo, 2012). To this end, the GLI1 antagonist, GANT-61, can reduce tumor formation by a KS-derived cell line. The sphere-forming efficiency, as well as the average volume of the formed spheres, were significantly decreased in GANT-61-treated cells, suggesting GLI1 inhibitors may act to attenuate tumor formation during KS initiation or progression. Immune evasion strategies, such as HLA class I downregulation is frequent during the progression of human tumors. In EBV-associated gastric cancer, latency I viral genes are often expressed (Deb Pal and Banerjee, 2015). Among the latency I viral genes, EBERs and $L M P 2 A$ are frequently detected in most gastric cancer samples, suggesting the virus expresses only cell context-adapted genes. It has been reported that LMP2A exploits the $\mathrm{HH}$ pathway by activating $\mathrm{SHH}$ signaling to induce HLA class Ia downregulation in gastric cancer cells (Deb Pal and Banerjee, 2015). LMP2Ainduced downmodulation or complete loss of HLA class Ia expression was specifically mediated by elevated GLI1 protein expression. Furthermore, inhibition of other important selfrenewal pathways such as Notch, WNT, or PI3K, in LMP2Aexpressing gastric cancer cells could not prevent HLA class I down-regulation, providing evidence in support of the hypothesis of cell-context and signaling-specific requirements by the virus (Deb Pal and Banerjee, 2015).

Overall, viruses can impinge on $\mathrm{HH}$ signaling in several ways. For example, EBV, HPV, HTLV, MCPyV, HBV, and HCV can trigger an autocrine $\mathrm{SHH}$ signaling loop to promote tumor development and stemness-associated programs. Instead, KSHV triggers Gli-mediated angiogenesis (VEGF) without involving $\mathrm{SHH}$. The hepatotropic $\mathrm{HCV}$ and $\mathrm{HBV}$ can cause chronic inflammation and turn on an inactive $\mathrm{HH}$ signaling in the healthy liver to promote fibrosis and HCC development. Another mode of action to perturb $\mathrm{HH}$ signaling is through alteration of Gli transcription as highlighted by HPV encoded E6 protein.

Autocrine stimulation plays an indispensable role in $\mathrm{HH}-$ mediated viral carcinogenesis, but its effect in the tumor microenvironment is still uncovered. Future studies are required to investigate how viruses utilize and relocate $\mathrm{HH}$ family members in infected cells to drive host cell machinery to carcinogenesis.

\section{NOTCH SIGNALING IN VIRAL ONCOGENESIS}

\section{Overview of the Notch Pathway}

Morgan (1917) observed a "notch" in the wings of a mutant Drosophila. It was subsequently found to be linked to a heterozygous deletion of a gene located on the chromosome $\mathrm{X}$, hence named Notch. The Notch signaling cascade is highly conserved from Drosophila to humans (Artavanis-Tsakonas et al., 1999), and consists of receptors, ligands, and intracellular proteins that transmit the signals to the nucleus. The four mammalian Notch receptors (Notch1-4) are large Type I transmembrane proteins. A furin-like convertase catalyzes the proteolytic maturation of Notch receptor pro-proteins in the Golgi apparatus (Logeat et al., 1998). The proteolysis generates two subunits connected by $\mathrm{Ca}^{2+}$-dependent ionic bonds: the Notch extracellular domain (NECD), consisting of multiple epidermal growth factor-like (EGF) repeats which mediate ligand 
binding, and the Notch intracellular domain (NICD), which is the transcriptionally active part of the molecule (Gordon et al., 2008; Yavropoulou and Yovos, 2014). NICD translocates to the nucleus where it binds Recombination signal binding protein for immunoglobulin kappa J region $(\mathrm{RBP}-\mathrm{J} \kappa)$ and, in cooperation with Mastermind-like 1 (MAML1), regulates the transcription of Notch target genes (Borggrefe and Oswald, 2009; Borggrefe et al., 2016; Roo and Staal, 2020). Interestingly, MAML1 is a versatile coactivator in other signaling pathways too. Indeed, it can serve as a Notch-independent transcriptional activator in the $\mathrm{HH}, \mathrm{Wnt} / \beta$-catenin and Hippo signaling pathways (Quaranta et al., 2017; Zema et al., 2020). The Notch pathway signaling can be "canonical" or "non-canonical," based on whether NICD interacts with RBP-Jא or not (Ayaz and Osborne, 2014). Canonical Notch ligands are also Type I transmembrane proteins and belong to the Delta/Serrate/LAG-2 (DSL) family of proteins. Considered to be the structural homologs to the Delta and Serrate ligands of Drosophila, mammalian Notch ligands are Delta-like proteins, named Dll1, Dll3, and Dll4, and Serrate homologs known as Jagged1 and Jagged2 (JAG1 and JAG2) (D'Souza et al., 2008; Kopan and Ilagan, 2009). Independently of Notch, Jagged 1 can induce intrinsic reverse signaling within the ligand-expressing cell as demonstrated in CC cells (Pelullo et al., 2019). One non-canonical Notch signaling example is the regulation of $\mathrm{Wnt} / \beta$-catenin signaling, which uses $\beta$-catenin as a transcriptional mediator (Sanders et al., 2009; Andersen et al., 2012).

Ligand-receptor interactions and transcription factor activity in Notch signaling play pivotal roles in a wide variety of differentiation processes, including regulation of cell-fate determination during tissue and cell development (Wang et al., 2015; Chandiran et al., 2018; Ferrandino et al., 2018b). Notch signaling affects proliferation, apoptosis, and cell differentiation (Grazioli et al., 2017). Moreover, active Notch signaling allows cells to maintain stem-cell-like features (Kopan, 2012; Kessler et al., 2015; Moriyama et al., 2018).

\section{Notch Signaling in Viral Oncogenesis}

Perturbing the Notch pathway can lead to the onset of various diseases, including cancer (Sjölund et al., 2005; Penton et al., 2012; Takebe et al., 2015; Krump and You, 2018). Coopting and dysregulating developmental pathways by oncogenic viruses also involve targeting Notch signaling components (Figure 2) (Krump and You, 2018). HBV proteins activate Notch signaling to stimulate uncontrolled cell proliferation, which then may lead to HCC (Mesri et al., 2014). The HBV encoded protein, $\mathrm{HBx}$, is one of the key viral factors capable of malignantly transforming infected cells. It upregulates Notch1 receptor, either through a direct interaction or through the p38 MAPK pathway, to promote HCC proliferation (Kong et al., 2016). This viral protein also stimulates Notch1-4 expression. The cytoplasmic Notch1 and the nuclear Notch4 correlate with $\mathrm{HBx}$ expression in HCC tissues (Gao et al., 2016). HBx-Dll4-Notch1 axis seems to have a critical role in regulating cell survival in HCC. Indeed, $\mathrm{HBx}$ mediates Dll4 upregulation, which increases Notch1 cleavage, thus activating Notch signaling (Kongkavitoon et al., 2016). Crosstalk between
HBx and JAG1 in HCC has also been documented. JAG1 is highly expressed in $\mathrm{HCC}$ tissues and is regulated by $\mathrm{HBx}$, further confirming an oncogenic role of the latter in activating Notch signaling (Gao et al., 2007). HBx involvement in HCC pathogenesis was further demonstrated by the discovery of a regulatory axis between this viral protein and miR3188. This oncogenic miRNA is overexpressed in HCC tissue and knocking-out miR-3188 using CRISPR/Cas9 de-repressed expression of its target (zinc fingers and homeoboxes 2) (ZHX2), a transcriptional repressor of Notch1. Thus, at least one way by which miR-3188 acts to induce Notch signaling and promote HCC pathogenesis is by negatively regulating ZHX2 (Zhou et al., 2017).

The EBV encoded nuclear protein EBNA2 is a biological equivalent of Notch1 (Zimber-Strobl and Strobl, 2001). Interestingly, this viral protein interacts with the same cellular repressor RBP-Jk as does NICD. EBNA2 and NICD, both have activating domains that interact with RBP-Jk transcriptional repressor, causing HDAC replacement leading to viral and cellular gene transcription (Strobl et al., 1997). Histone acetyltransferases (HATs), such as P300/CBP-associated factor (PCAF), also interact with EBNA2 and NICD in the context of gene transactivation. Constitutive activation of Notch1-4 in different cell types can lead to tumorigenesis (Aster et al., 2017; Tottone et al., 2019). Since Notch1 signaling is associated with cancer, it is significant that EBNA2 can hijack components of this pathway to immortalize and transform B cells into lymphoblastoid cell lines (LCLs) (Tierney et al., 2015). In a reciprocal experiment, Strobl et al. (2000) have shown that activated Notch1 (N1ICD) can substitute for some EBNA2 functions. Specifically, they observed that in stably transfected Burkitt lymphoma (BL) cell lines carrying EBNA2-deletion, N1ICD was able to induce expression of some but not all EBNA2-inducible genes, such as $c-m y c, C D 21$, and $L M P 2 A$, but not LMP1 or CD23 (Strobl et al., 2000). These observations have led to the hypothesis that in those EBV associated cancers where EBNA2 is not expressed, NICD signaling can play a very critical role in transformation (Höfelmayr et al., 2001; Zimber-Strobl and Strobl, 2001; Chiara et al., 2016). A previous study showed that EBV-immortalized LCLs are characterized by high levels of telomere-specific reverse transcriptase (TERT), a catalytic component of telomerase, which prevented the switch from latency to lytic cycle activation of EBV (Giunco et al., 2015). The underlying mechanism involved activation of Notch2, which in turn, induced the Basic Leucine Zipper ATF-like (BATF) transcription factor. BATF negatively regulated BZLF1, the master regulator of the EBV lytic cycle, thereby preserving the latent state of the virus and survival of the infected B cells (Giunco et al., 2015).

KSHV can also subvert Notch signaling to promote survival of KSHV-infected primary B cells (Lan et al., 2006). One of its latent proteins, LANA, interacts with the tumor suppressor Sel10-mediated ubiquitin-proteasome pathway, which negatively regulates NICD. A study showed that LANA sequesters Sel10 by forming a complex in primary effusion lymphoma (PEL) cells (Lan et al., 2007). This complex prevents Sel10-NICD interactions, resulting in stabilized NICD, increased cell 


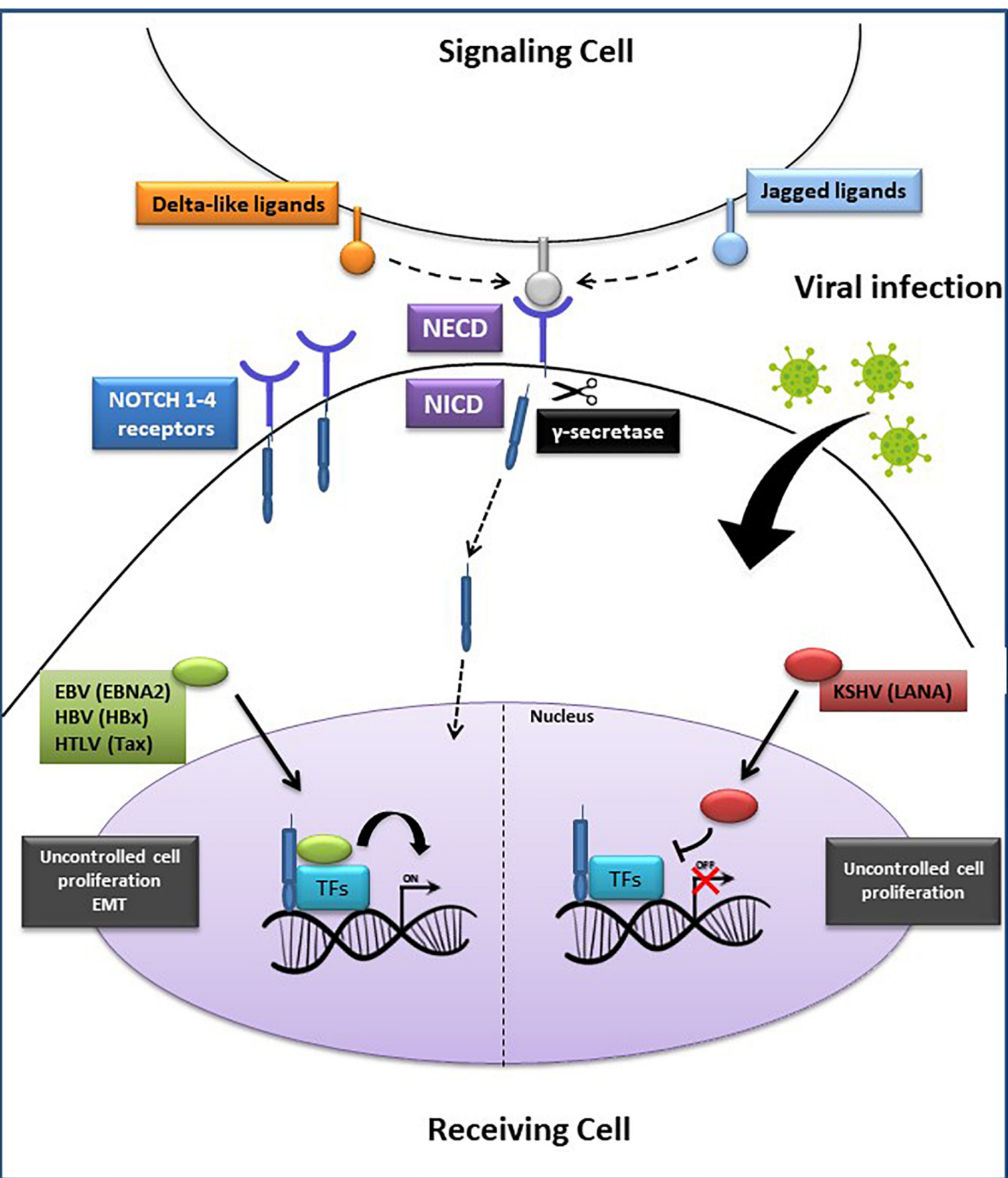

FIGURE 2 | Oncogenic viruses exploit the Notch signaling pathway. Notch ligands (Delta-like or Jagged) on the signaling cell bind to Notch 1-4 receptors on the receiving cell, generating the Notch extracellular domain (NECD) and the Notch intracellular domain (NICD). Subsequently, the proteolytic cleavage of NECD by $\gamma$-secretase, generates NICD translocation into the nucleus where it binds with the transcription factors (TFs): recombining binding protein Jk (RBP-JK), cAMP response element-binding protein (CREB), forming an active complex that regulates the transcription of Notch target genes. On the (Left side): EBNA2, HBx and Tax proteins encoded by EBV, HBV, and HTLV, respectively, promote cell proliferation or epithelial-mesenchymal transition (EMT) through a direct interaction with the transcription complex, leading to tumorigenesis. On the (Right side): LANA protein, encoded by KSHV, prevents the activation of the transcription complex, resulting in an accumulation of NICD and increased cancer cells proliferation. EBV, Epstein Barr Virus; HBV, Hepatitis B Virus; HTLV-1, Human T Lymphocyte Virus-1; KSHV, Kaposi's Sarcoma-associated Herpesvirus.

proliferation and angiogenesis. Moreover, KSHV-encoded replication and transcription activator (RTA) induced JAG1 expression, thereby activating Notch signaling. This leads to inhibition of lytic reactivation in a pro-lytic tumor microenvironment, maintaining the balance between lytic and latent state in KSHV infected cells, and ultimately resulting in virus-immune escape and persistence in the host (Li et al., 2016).

A recent study showed that in HPV-related CC, the Notch pathway is indeed affected by aberrant mutations, amplifications, and deletions (Yang et al., 2020). A copy number variation 
analysis (CNV) was performed in $282 \mathrm{CC}$ patients and among the affected genes, they found amplifications of $4 \mathrm{q} 34.1$ F-Box And WD Repeat Domain Containing 7 (FBXW7) and of 1p36.11 hairy and enhancer of split-1 (HES)2/3/4/5 tumor suppressor genes belonging to the Notch pathway. Interestingly, this was associated with significantly improved overall survival (OS) of these patients. In spite of several important limitations, such as the lack of information about HPV-type associated with the aberrant amplification of the two tumor suppressor genes of the Notch pathway, this study provides new insights for the prognosis of CC patients, based on tumor cell methylation signatures.

Notch signaling is exploited by the HTLV-1 encoded Tax1. This viral transcription factor activates Notch1 and prolongs the half-life of NICD, in ATL cells (Cheng et al., 2019). Tax1 formed a ternary complex with NICD, and RBP-J火, thus promoting cell proliferation and tumor progression. Another study showed that HTLV-1-encoded Tax induced JAG1 in most ATL cell lines, through the transcription factor NF-kB (Bellon et al., 2018). The ATL cell lines which did not express high levels of JAG1 were investigated for the presence of posttranscriptional inhibitory mechanisms mediated by miRNAs. The lack of JAG1 protein expression in these cell lines was due to high expression of miR-124a, which directly binds to the 3'UTR of JAG1 mRNA. Furthermore, in most ATL patients with high JAG1 expression, miR-124a levels were low. Interestingly, STAT3, and NFATC1 were also highly expressed. Remarkably, they are both miR-124a targets. The absence of miR-124a in ATL might enhance JAG1 and Notch1 signaling pathway sustained by constitutive expression of STAT3 and NFATC1. Therefore, inhibiting JAG1 could be a promising therapeutic strategy in ATL (Bellon et al., 2018).

Another virus that perturbs the Notch pathway is $\mathrm{HCV}$. The virus encoded non-structural protein 3 (NS3) is essential for its replication and contributes to viral induced HCC (Iwai et al., 2011). The Yeast two-hybrid screening and coimmunoprecipitation assays in mammalian cells, showed that Snf2-related CBP activator protein (SRCAP) interacted with NS3 and both proteins activated the Hes1 promoter, a downstream target of the Notch pathway. Thus, HCV NS3 together with SRCAP and a SRCAP-resembling protein, p400, activated the Notch signaling pathway. A more recent article has demonstrated report showed that Notch signaling and CD4 T helper 22 (Th22) cells are involved in chronic HCV pathogenesis (Jiang B.C. et al., 2017). Specifically, the Notch interaction with aryl hydrocarbon receptor (AhR) induced IL-22 production by Th22 cells, thus favoring persistent $\mathrm{HCV}$ infection. Another study reported that Notch 1 and -2 enhanced regulatory $T$ cells (Tregs) and Th17 cell functions thus facilitating HCV infection (Qin et al., 2017). These studies suggest that inhibiting $\gamma$-secretase and thus Notch activation, might enhance immune surveillance against chronic HCV infection by down-regulating the production of IL-22, IL-17, as well as Treg-mediated immune tolerance in patients with chronic HCV.

Since oncogenic viruses co-opt Notch pathway components to sustain their survival leading to tumor progression, the development of inhibitors to block virus-Notch interactions could become a valuable therapeutic approach. To this end, utilizing monoclonal antibodies, drugs targeting Notch receptors, $\gamma$-secretase inhibitors (GSI), or small molecules that disrupt the interaction between Notch and RBP-Jk, RBPJ INhibitor1 (RIN1), provide novel therapeutic avenues to pursue. For instance, monoclonal antibodies (mAbs) specifically targeting Notch1 reduced stemness of breast cancer cells (Sharma et al., 2012), whilst blocking antibodies against JAG1 in colorectal cancer (CRC) patients provided therapeutic benefits with low intestinal toxicity (López-Arribillaga et al., 2018). Enoticumab, an anti-Dll4 mAb, administered to patients with advanced solid tumors, inhibited the growth of these tumors and in ovarian cancer (OC), in a dose-dependent manner (Chiorean et al., 2015). RIN1 inhibited RBP-Jk transcription and interaction with NICD, thereby reducing proliferation in T-ALL and mantle cell lymphoma (MCL) cell lines (Hurtado et al., 2019). Focusing on viral-Notch interactions, it has been shown that H1N1 influenza virus challenge in mice increased Notch ligand Dll1 expression on macrophages, dependent on retinoic acid-inducible gene-I (RIGI), which in turn induced the type I IFN pathway. Inhibiting $\gamma$-secretase during viral infection resulted in decreased IFN $\gamma$ production, increased $\mathrm{H} 1 \mathrm{~N} 1$ load and acute inflammation in mouse lungs (Ito et al., 2011). Treating primary and immortalized KSHV cells with the GSI, LY-411,575 induced apoptosis in these cells, revealing a therapeutic alternative for patients with KSHV related diseases (Curry et al., 2005). Additionally, given that Notch 2 plays an important role in maintaining EBV latency, the use of GSIs has been proposed as a therapeutic strategy for EBV associated lymphomas (Giunco et al., 2015).

With some exceptions mentioned above, such as in the case of H1N1, in which inhibiting the Notch pathway with GSI led to adverse effects in mice, the Notch inhibitors have the potential to be therapeutically implemented with necessary precautions to interrupt the Notch pathway activation by viral proteins.

\section{Viruses Exploit Notch Signaling to Escape Immune Responses}

Immune escape by tumor cells is a fascinating phenomenon. Indeed, tumor development is, quintessentially, a failure of the immune system to recognize and eliminate the cells that have emancipated themselves from cell cycle control and have gone awry (Prendergast, 2008). The immune evasion is achieved in several different ways. For instance, downregulation of HLA molecules on the tumor cell surface, upregulation of inhibitory immune checkpoint proteins, alteration of tumor cell death pathways, or an increase in immunoregulatory and immunosuppressive cytokines within the tumor microenvironment (TME) are some examples how tumor cells become invisible to the immune control.

A paradigmatic example of how Notch pathway activation could be central to immune suppression is provided by EBV associated Hodgkin's lymphoma (HL). This tumor is characterized by increased infiltration of regulatory $\mathrm{T}$ (Treg) cells. The characteristic Reed Sternberg (RS) cells, which represent the HL tumor component, highly express Notch1 and Notch2 (Jundt et al., 2002). Interestingly, the same cells also produce high levels of CCL22 (Ishida et al., 2006) and this chemokine is 
important for recruiting Tregs into the TME. It has also been shown that another chemokine, CCL20, is upregulated in HL by the EBV-encoded nuclear protein, EBNA1. Just as CCL22, CCL20 is also critical for the increased numbers of Tregs see in HL. Given that Notch signaling can significantly influence cytokine and chemokine profiles in tumor cells to alter the tumor landscape (Colombo et al., 2018), it will be important to investigate if increased Notch 1 and 2 expression in RS cells might affect CCL22 and CCL20 expression to augment regulatory T cell recruitment in $\mathrm{HL}$.

Non-coding RNAs, especially miRNAs, play a significant role in helping tumors escape immune control by negatively regulating critical immunomodulatory genes (Li et al., 2021; Mondal et al., 2021). MiR-346 targets transport associated protein 1 (TAP1) and reduces transport of peptides into the endoplasmic reticulum for binding to HLA class I molecules (Bartoszewski et al., 2011). MiRNAs are also involved in immune checkpoint regulation. Recently, we have shown that EBV-encoded EBNA2 can induce PD-L1 expression in lymphoma cells (Trivedi et al., 2018; Anastasiadou et al., 2019). The increase in PD-L1 was due to a decrease in miR-34a. Interestingly, miR-34a also targets Notch (Kang et al., 2013). Thus, it seems that EBNA2, in addition to acting as a functional homolog of NICD, may also keep Notch expression and activity high by downregulating miR-34a. It will also be important to examine whether Notch and PD-L1 expression are positively correlated.

In HBV associated HCC, the metastatic event characterized by the portal vein tumor thrombus (PVTT), is common in over a third of patients. The positive correlation between the presence of HBV and PVTT has been established (Yang et al., 2012). These PVTT symptoms of HCC were also positively correlated with $\mathrm{TGFb}$, where an increase in $\mathrm{TGFb}$ led to downregulation of miR-34a. Strikingly, CCL22 was shown to be an authentic target of miR-34a. Thus, TGFb-mediated miR34 a downregulation in HCC led to an increase in CCL22 and, consequently, to regulatory $\mathrm{T}$ cell recruitment to create an immunosuppressive tumor environment (Figure 3). The role of $\mathrm{HBV}$ in PVTT development is clear because over $82 \%$ of $\mathrm{HBV}$ positive HCC patients developed PVTT, compared with only $14 \%$ HBV negative HCC patients (Yang et al., 2012). As mentioned above, since miR-34a downregulates Notch, it will be critical to establish the link between high CCL22 and Notch expression and how Notch signaling may play an immunosuppressive role in HBV associated HCC. Furthermore, several studies have shown that aberrant activation of NICD in T cells may lead to T-cell acute lymphoblastic leukemia (T-ALL) confirming the importance of the Notch pathway in the progression of immune system-related malignancies (Ferrando, 2009; Bernasconi-Elias et al., 2016; Ferrandino et al., 2018a).

It is remarkable how diverse viral proteins, such as EBNA2, LANA and Tax1, are all able to deregulate the same effector of the Notch pathway, NICD, leading to cell transformation, latency maintenance, proliferation, angiogenesis and tumor progression. HBx and NS3 viral proteins interact with other activators of the Notch pathway, such as JAG1, Dll4, and SRCAP, respectively, to favor HCC progression. Continued investigation will enable a deeper understanding of how viral proteins interact with the
Notch signaling to take advantage of host cells and escape immune surveillance. The knowledge of these interactions is an important step for the development of targeted therapies in virus-associated cancer patients.

\section{WNT SIGNALING IN VIRAL ONCOGENESIS}

\section{Overview of WNT Signaling}

WNT signaling is involved in cell proliferation, cell polarity, and cell fate determination during embryonic development and tissue homeostasis. Evolutionarily, the WNT signaling pathway is highly conserved. Under normal or homeostatic conditions, in the absence of WNT ligands, the signaling is maintained in an "off" state (Figure 4A). Under this condition, b-catenin that is not membrane-associated is sequestered in a cytosolic complex together with Auxin, adenomatous polyposis coli (APC), casein kinase I (CKI), and glycogen synthase kinase (GSK) 3b. Within this complex, b-catenin is sequentially phosphorylated by CKI and GSK3b, which primes b-catenin for ubiquitination and proteasomal degradation (Stamos and Weis, 2013). This process of active degradation prevents b-catenin from translocating to the nucleus. In the absence of nuclear b-catenin, WNT target gene transcription is repressed when Transcription Factor (TCF) binds to WNT gene promoters in association with transcriptional corepressors, such as TLE1 (Transducin-Like Enhancer of Split1).

WNT signaling is activated when secreted WNT proteins bind to the seven-pass transmembrane receptor, Frizzled (FZD), together with its co-receptor, low-density lipoprotein receptor related protein (LRP) 6, or to the closely related, LRP5. Following WNT binding, the FZD-LRP6 receptor complex associates with the Disheveled segment polarity protein (Dvl), a scaffold protein that facilitates LRP6 phosphorylation and subsequent recruitment of Axin way from APC and GSK3b to form the FZD-WNT-LRP6-Dvl-Axin multiprotein complex (Komiya and Habas, 2008). As the interactions of Axin with APC and GSK3b are diminished, the complex loses its ability to bind to b-catenin and mediate its degradation. As a result, b-catenin accumulates in the cytosol and, ultimately, translocates to the nucleus, where it successfully out-competes TLE for binding to TCF and activates WNT target gene transcription (Figure 4B).

WNT signaling regulation is complex. Many components of this pathway are, themselves, positively or negatively regulated through the WNT pathway. Through their co-evolution with the human immune system, oncogenic viruses have devised means either to down-regulate inhibitory WNT proteins, such as Dickkopf WNT signaling pathway inhibitor1 (DKK1) (Niida et al., 2004) (Figure 4C) or reinforce positive WNT signaling networks (Figure 4D) to promote cellular transformation and tumorigenesis.

\section{Oncogenic Hepatitis Viruses Modulate WNT Signaling}

Oncogenic HBV and HCV infect cells of the liver causing hepatic inflammation, fibrosis, cirrhosis, and, ultimately, HCC. The 


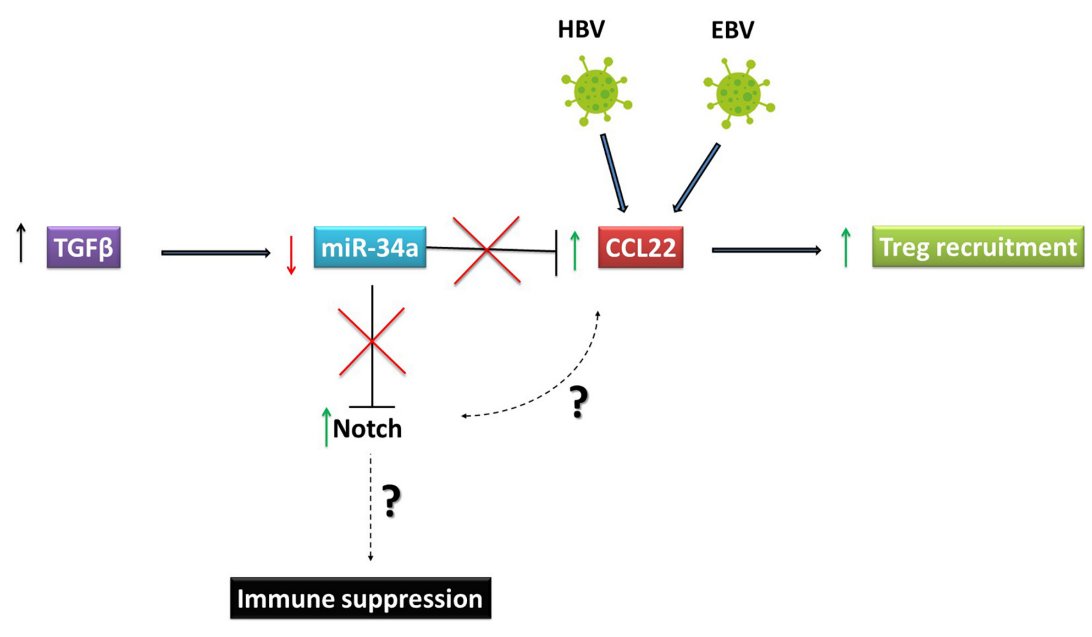

FIGURE 3 | Oncoviruses exploit Notch signaling to escape immune responses. Reed Sternberg cells, which represent the Hodgkin's Lymphoma tumor component are high expressers of Notch 1 and 2 and produce high levels of CCL22. This chemokine is important for recruiting regulatory $T$ (Treg) cells to the tumor microenvironment. In HBV associated HCC, PVTT symptoms are positively correlated with TGF $\beta$. The increase in TGF $\beta$ led to downregulation of miR-34a, which targets CCL22. Downregulation of miR-34a induced by TGF $\beta$ in HCC led to an increase in CCL22 and consequently in regulatory T cell recruitment to create an immunosuppressive tumor environment. HBV, Hepatitis B virusVirus; EBV, Epstein Barr Virus; HCC, Hepatocellular Carcinoma.

progression from initial infection to HCC is a lengthy, complex, multi-step process. HBV and HCV are vastly different - HBV is a partially double-stranded DNA virus (Liang, 2009), while $\mathrm{HCV}$ is a positive-sense, single-strand RNA virus (Dubuisson and Cosset, 2014)- making it unsurprising that the molecular mechanisms by which they induce tumorigenesis also differ. However, evidence suggesting that both HBV and HCV hijack components of the WNT signaling pathway underscores the importance of subverting WNT signaling in HCC.

\section{Oncogenic Viruses Subvert WNT Signaling Through Multiple Mechanisms}

Mutations in CTNNB1, the b-catenin encoding gene, is associated with HCC (Javanmard et al., 2020). However, genomic instability caused by HBV infection is more commonly associated with inactivating mutations in AXIN1 leading to $\mathrm{HCC}$ ( $\mathrm{Li}$ et al., 2013). The HBV genome consists of four overlapping genes encoding surface antigens ( $S$ gene), core proteins ( $C$ gene), HBV polymerase ( $P$ gene), and the HBx protein $(X$ gene). The polymerase lacks proofreading activity, so HBV mutations occur with greater frequency than other DNA viruses. Frequently, these mutations are associated with activation of WNT signaling. Mutations in the core promoter, overlapping with the $X$ gene, have also been described. The resulting single or combination point mutations in $\mathrm{HBx}$, which is thought to act as a promiscuous transcription factor, also upregulated WNT signaling (Chen et al., 2016). Overexpressing HBx mutants in human hepatocyte cell lines increased phosphorylated GSK3b, in the absence of increased total GSK3b. This suggests $\mathrm{HBx}$ mutants may be acting upstream of GSK3b to regulate its phosphorylation and subsequent inactivation, although whether this occurs as a result of Srk or Erk kinase activation or through its interaction with $A P C$, remains to be fully elucidated. Functionally, cells expressing
HBx mutants showed greater proliferation and migration, which could be abrogated by co-expressing siRNA to b-catenin. HBx mutant cells showed increased expression of cytoplasmic and nuclear b-catenin and, importantly, HCC patient samples with combination mutations also showed high levels of cytoplasmic b-catenin as well as evidence of nuclear accumulation. Welldocumented WNT pathway proteins were also upregulated, including c-Myc, Connective Tissue Growth Factor (CTGF), Cyclin D1, and WISP2, in mutant HBx-expressing cells, suggesting these mutations act at multiple levels to increase WNT signaling (Figures 4C,D).

Hepatitis B Virus has been shown to increase WNT signaling by upregulating its activating ligand Frizzled7 (FZD7) (Merle et al., 2004). Additional HBV proteins are also thought to participate in this activating pathway. The pre-core protein 22 (p22) is further processed to p17, also known as HBeAg, before being secreted by infected cells (Tran et al., 2020). A human HCC cell line, Huh7, when transfected with p22 upregulated FZD7 to a greater extent than HBx (Tran et al., 2020). Expressing p22 in cell lines without or with known mutations in APC or b-catenin resulted in a synergistic increase in TCF/CTNNB1 transcription that could be reversed with co-expression of DNTCF4. FZD7 is not the only HBx-induced ligand implicated in activating WNT signaling. Expressing $\mathrm{HBx}$ in a normal liver cell line upregulated N1ICD and increased expression of FZD10, but not FZD7 (Sun et al., 2014). Cyclin D1 and b-catenin levels were also upregulated, along with increased cytosolic and nuclear b-catenin. WNT signaling was diminished in cells treated with a Notch inhibitor, or in which NOTCH1 was knocked down using siRNA approaches. However, delivering siFZD10 to cells had no effect on NOTCH1 expression, placing NOTCH1 upstream of WNT pathway activation in these cells. Thus, WNT signaling is likely activated by $\mathrm{HBx}$ through different mechanisms to promote HCC. 


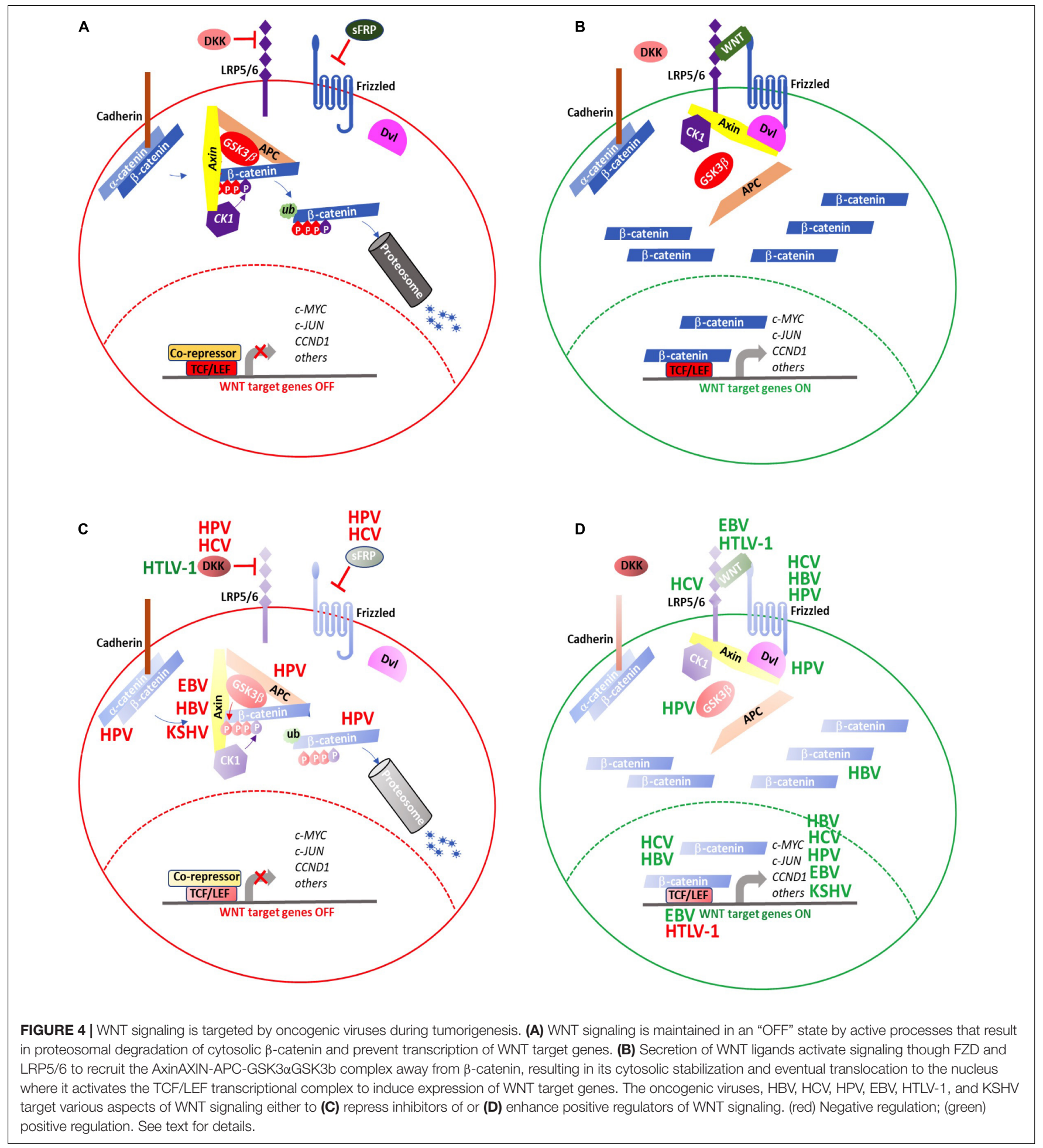

In addition to HBV, HCV can also promote HCC by instilling extended, sequential changes to the liver that occur over time. The HCV genome encodes structural proteins like core, E1, E2, p70 and non-structural proteins namely NS1, NS2, NS3, NS4A, NS4B, NS5A, and NS5B (Mahmoudvand et al., 2019). The HCV core, NS3, NS5A, and NS5B proteins contribute to HCC by interacting with and modulating key host cellular functions, such as cell cycle, proliferation, and apoptosis. When core protein Type 1B or NS4B was expressed in Huh7 cells, or in the normal liver L02 cell line, increased nuclear b-catenin was observed upon activation of WNT3a (Jiang X.H. et al., 2017). This was accompanied by increased expression of MYC, 
WNT1, and CCND1 in Huh7, but not in L02 cells, suggesting these $\mathrm{HCV}$ proteins increase WNT signaling to directly affect WNT-mediated cellular processes (Jiang X.H. et al., 2017). The $\mathrm{HCV}$ core protein has been shown to inactivate GSK3b, further promoting $\mathrm{WNT} / \mathrm{b}$-catenin activity. In the nucleus, b-catenin complexes with TCF to activate downstream targets such as MYC, CCND1, and WISP2, to enhance cell-cycle progression and cell proliferation (Mahmoudvand et al., 2019). HCV core protein can also act positively to increase $\mathrm{WNT} / \mathrm{b}$-catenin signaling by upregulating expression of LRP5/6 and FZD receptors. Increased signaling through these receptors releases b-catenin from E-cadherin complexes, facilitating its translocation to the nucleus, and subsequent transcriptional activity (Figures 4C,D) (Mahmoudvand et al., 2019). To further complicate the predicted effects of core proteins on patient disease progression, Aicher et al. (2018) used HEK293 and Huh7.5 cells to demonstrate core protein sequence-specific effects on the expression of b-catenin and its transcriptional targets, based on the differences in $\mathrm{HCV}$ substrains obtained from clinical isolates. Thus, although it is clear that HCV co-opts WNT signaling pathways to promote $\mathrm{HCC}$, it is possible this occurs in a substrain-specific manner, at least with regard to core protein effects.

Hepatitis $\mathrm{C}$ virus infection is also associated with promoter hypermethylation of the WNT pathway inhibitors, Secreted Frizzled-related Protein 2 gene (SFRP2) and DKK1, leading to WNT/b-catenin activation. Studies by Umer et al. (2014) suggest SFRP2 and DKK1 hypermethylation may occur early following HCV infection and may lead to HCC via a multi-step process. In this study, the authors utilized bisulfite sequencing to analyze liver biopsies from patients with chronic hepatitis, liver cirrhosis, and HCC. Compared to normal liver samples, SRFP2 and DKK1 showed significant hypermethylation in $\mathrm{HCV}$-infected patients. Although no differences in DKK1 methylation was noted between the different conditions, there was a progressive increase in SFRP2 promoter methylation with normal liver $<$ chronic hepatitis < liver cirrhosis < HCC (Umer et al., 2014). These findings are consistent with those of Eldeeb et al. (2020) which showed that in HCV-infected patients with liver cirrhosis, with or without corresponding HCC, DKK1 expression was significantly decreased. Thus, evaluating promoter methylation status, especially for SFRP2, or DKK1 protein levels may prove to be useful for monitoring disease progression following $\mathrm{HCV}$ infection (Figure 4C).

In an interesting study, Zhang et al. (2012) examined how $\mathrm{HCV}$ infection intersects with miRNA expression. HCV-infected Huh7 cells expressed higher levels of the pro-inflammatory miRNA, miR-155, via an NF-kB-mediated pathway. This resulted in greater accumulation of nuclear b-catenin, along with increased expression of cMYC, Cyclin D1, and survivin. Increased miR-155 did not appear to affect GSK3b or AXIN1 signaling. Functionally, high miR-155 expression led to a block in apoptosis and increased cellular proliferatio in vitro and increased tumorigenesis in nude mice. $\mathrm{HCV}$ core protein can also act positively to increase $\mathrm{WNT} / \mathrm{b}$-catenin signaling by upregulating expression of LRP5/6 and FZD receptors. Increased signaling through these receptors releases b-catenin from E-cadherin complexes, facilitating its translocation to the nucleus and subsequent transcriptional activity. Taken together, these data point to additional direct and indirect means by which HCV promotes HCC through dysregulated WNT signaling.

\section{WNT Signaling Is Dysregulated Following Infection With HPV or EBV}

HPV codes for multiple viral proteins with critical functions in viral infection, integration and replication. Its capacity to transform human epithelial cells are ascribed to its E6 and E7 proteins, and this includes promoting b-catenin translocation to the nucleus where it acts transcriptionally, to facilitate tumorigenesis. Unlike the actions of $\mathrm{HCV}$ and $\mathrm{HBV}$, HPV infection rarely causes mutations in CTNNB1 or AXIN1. However, HPV can activate components of the WNT signaling pathway to facilitate cellular transformation. Microarray gene analysis shows HPV infection induces multiple genes, including upregulation of WNT related proteins (FragosoOntiveros et al., 2012). It has also been shown that in some cervical and oropharyngeal squamous cell cancers, HPV infection reduces the amount of membrane-associated b-catenin while, at the same time, it increases its cytosolic and nuclear accumulation (Rodríguez-Sastre et al., 2005). HPV can further act to increase GSK3b phosphorylation, which can further attenuate its inhibitory action on WNT signaling (Figures 4C,D) (Rath et al., 2015).

Akin to HCV, HPV can also modulate miRNAs expression to affect WNT signaling. Mo et al. (2015) showed HPV can increase expression of miRNAs, the targets of which may repress WNT signaling only during early stages of CC development. The authors suggest that the differential regulation of AXIN2, DVL3, and LEF1 by miR-622, miR-920, and miR-507, respectively, may act to stabilize or increase WNT signaling at later stages of cancer progression (Mo et al., 2015). However, additional functional studies are needed to confirm how differences in miRNA expression contribute to cellular transformation caused by HPV.

Epigenetic changes that modulate the WNT pathway have been observed as a result of HPV infection and, frequently, the WNT inhibitory genes are targeted. For instance, in some HPV associated OC, increased APC and SFRP3 promoter methylation have been observed (Al-Shabanah et al., 2014). In HPV positive CC, hypermethylated SFRP 2 and DKK3 promoters have also been reported (van der Meide et al., 2011).

Studies suggest HPV can also affect other proteins that crosstalk with the WNT pathway. LRG5 is a G-protein coupled receptor. Chen Q. et al. (2014) demonstrated that, as HPV-induced CC progressed, increased LGR5 expression could be detected via immunohistochemistry. Furthermore, in vitro reporter assays indicated that LGR5 activates WNT signaling by upregulating $\mathrm{c}-\mathrm{myc}$, cyclin D1, and b-catenin to increase cell cycle progression and drive proliferation (Chen Q. et al., 2014). HPV can also modulate less well-known WNT signaling partners like, FOXM1, a novel component of WNT signaling (Chen P.M. et al., 2014). Patients diagnosed with lung or oral cancer showed worse overall and relapse-free survival compared to patients who lacked a demonstrated interaction between b-catenin and FOXM1 (Chen P.M. 
et al., 2014). Finally, Lichtig et al. (2010) provide evidence that HPV infection may even alter b-catenin proteasomal degradation and this may require E6/E6AP. Thus, HPV infection can successfully promote WNT-mediated epithelial cell transformation through multiple mechanisms to directly or indirectly activate the WNT pathway.

EBV is yet another oncovirus that targets the WNT signaling pathway to transform epithelial cells. Studies using telomeraseimmortalized normal oral keratinocytes (NOKs) showed that EBV infection led to epigenetic reprogramming, CpG hypermethylation, and delayed responsiveness to differentiation cues (Birdwell et al., 2014). Using the same system, Birdwell et al. (2018) further investigated the effects of EBV infection on cellular transformation and discovered that the invasive phenotype acquired by NOKs persisted, even after the viral loss. LEF and WNT5a, both of which are elevated in NPC, were increased in EBV-infected NOKs. LEF and WNT5a expression remained high for more than 20 passages, after EBV viral expression was no longer detected. Forced expression of LEF and WNT5a enhanced the invasive capacity of NOKs, while knocking down $L E F 1$ reduced their invasiveness, even in the presence of WNT5a expression. The data suggest that EBV may provide a selective advantage to infected cells, with LEF1 contributing to their metastatic potential (Birdwell et al., 2018).

The tumor suppressor, DACT2, is expressed in various healthy tissues and is a methylation target in some cancers (Zhang et al., 2018). Zhang et al. (2018) demonstrated this WNT regulator was also the target of hypermethylation in EBVrelated NPC. Treating NPC cells with the DNA methyltransferase inhibitor, 5-aza-2'-deoxycytidine, restored DACT2 expression to normal levels. Ectopic expression of DACT2 in NPCs also reduced proliferation, migration, and invasion, and induced G2/M arrest by blocking b-catenin/Cdc25 activity (Zhang et al., 2018). Restoring DACT2 expression sensitized NPC cells to the cytotoxic actions of paclitaxel and 5FU, but not to cisplatin, suggesting DACT2 may be an additional means of modulating the WNT pathway in NPC.

EBV also affects miRNAs to regulate WNT signaling in NPC. The EBV encoded miR-BART22 can induce the cellular miR4721 through a PI3K/AKT/cMYC/cJUN/Sp1 mediated pathway. GSK3b is a direct target of miR-4721 and in clinical samples, low GSK3b expression correlates with high miR-4721 levels (Tang et al., 2020). Increased miR-4721 further correlates with increased nuclear b-catenin accumulation and greater CCND1 and MYC expression (Tang et al., 2020). Thus, as with other oncogenic viruses, EBV promotes tumorigenesis by subverting WNT signaling through direct and indirect mechanisms.

\section{Immune Cells as Targets of Oncogenic Viral Transformation}

HTLV-1 infection is the underlying cause of ATL in a significant proportion of infected individuals. The virus expresses several proteins known to facilitate leukemic transformation, including the basic leucine zipper (bZIP) factor, HBZ, and the Tax protein; however, they can have opposing effects on WNT signaling. For instance, HBZ and Tax, both can interact with the WNT pathway through a related protein, DAPLE (disheveled-associated protein with a high frequency of leucine residues) (Ma et al., 2013). In the presence of DAPLE, Tax can activate canonical WNT signaling while HBZ suppresses this activation. One way by which HBZ inhibits canonical WNT signaling is by impairing LEF DNAbinding. HBZ can also enhance TGFb-mediated transcription of WNT5A to antagonize the canonical WNT pathway (Ma et al., 2013). Knocking down WNT5A in HTLV-1-infected cells repressed cellular proliferation and migration, confirming the contribution of WNT signaling to the leukemic process. These contradictory findings suggest that it may be the balance of Tax and HBZ expression following HTLV-1 infection that ultimately tips the scale in favor of leukemogenesis (Figures 4C,D).

To highlight the complex interactions between HTLV-1 and the WNT pathway, it is interesting to note that while Tax functions to promote HTLV-1 viral replication, TCF1 and LEF1 both interact with Tax to attenuate Tax-dependent viral expression and activation of NF-kB and AP-1 (Ma et al., 2015). In contrast, both TCF and LEF are downregulated in activated T cells. Ma et al. (2015) provide in vivo data supporting this yin and yang between TCF/LEF expression and viral load using Japanese macaques as a model system. In animals infected with the closely related, Simian T Lymphocyte Virus-1 (STLV-1), a negative correlation between the STLV-1 proviral load and TCF/LEF1 expression was observed in T cells (Ma et al., 2015).

Among its symptoms, patients with severe cases of ATL may exhibit hypercalcemia, bone loss, and bone lesions that are associated with osteoclast-mediated bone resorption (Xiang et al., 2019). One key mediator of these processes is the WNT inhibitor, DKK1, which has been identified as a key regulator of hypercalcemia and bone loss (Colditz et al., 2019). In keeping with the role of Tax protein in modulating WNT signaling, it represses DKK1 in HTLV-1 infected cells (Polakowski et al., 2010). However, DKK1 has also been associated with bone lesions in Multiple Myeloma (Fujita and Janz, 2007). Polakowski et al. (2010) found that HBZ nuclear localization can modulate transcription by binding to p300/CBP transcriptional co-activators. Microarray analysis of cells expressing wildtype or mutant $\mathrm{HBZ}$ revealed transcriptional upregulation of $D K K 1$, which was attenuated following siRNA knock-down of p300/CBP. Forced HBZ expression in T cells uninfected with HTLV-1 caused de novo expression of DKK1, while expressing HBZ in HTLV-1-infected $\mathrm{T}$ cells increased its expression (Polakowski et al., 2010). These data are consistent with another study that examined mechanisms of osteolytic bone lesions. Elevated serum levels of DKK1 has also been reported in a mouse model of ATL that expresses HBZ from a granzyme promoter (Esser et al., 2017).

Finally, the $\gamma$-herpesvirus KSHV, can target components of the WNT signaling pathway in B cells to promote tumorigenesis (Fujimuro et al., 2003). The LANA protein in KSHV shares homology with AXIN and has been shown to bind GSK3b (Fujimuro and Hayward, 2003). Furthermore, it was shown that interaction with LANA could draw GSK3b away from the inhibitory complex that keeps b-catenin inactive in the cytosol and allows for its nuclear translocation and subsequent transcription of WNT target genes. 


\section{Oncogenic viruses}

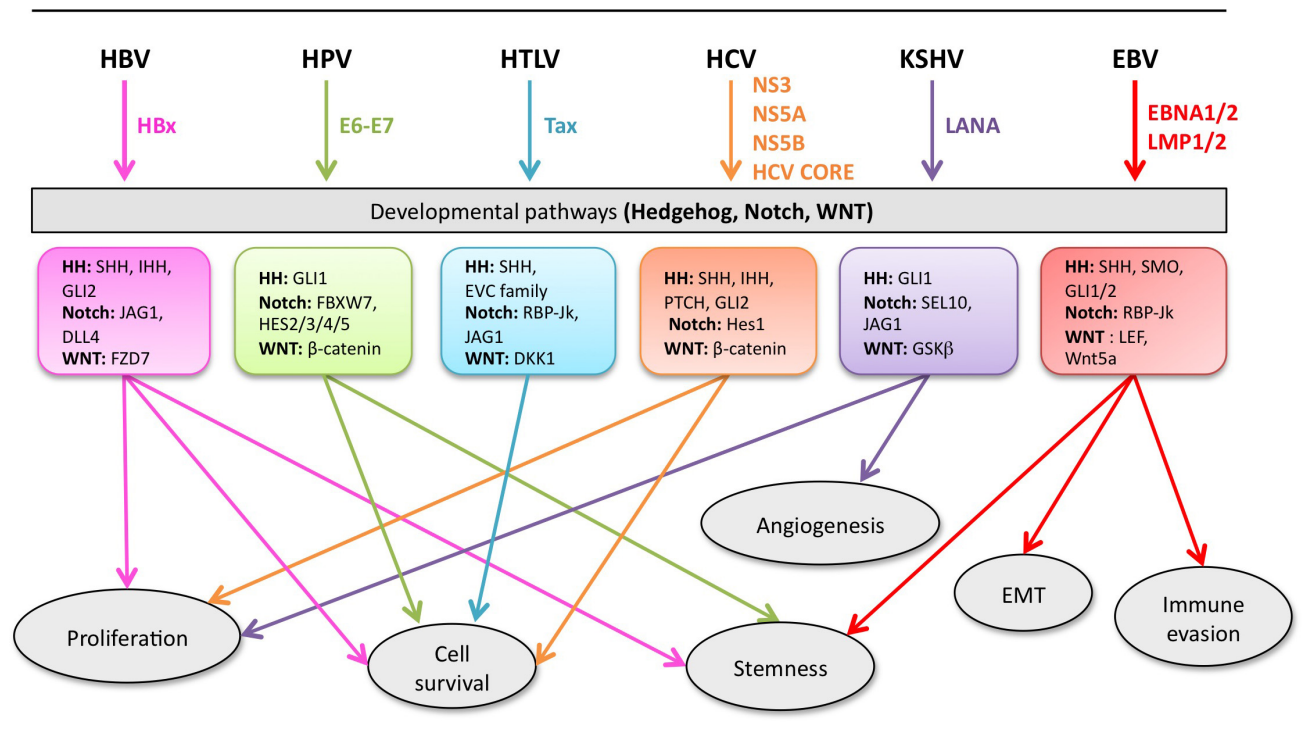

FIGURE 5 | Developmental pathways co-opted during viral oncogenesis. Viruses deregulate developmental signaling to support malignant transformation and disease progression, via a variety of mechanisms, including (but not limited) to, increased proliferation and cell survival, induced stemness to improve fitness of the cancer cells, accrued neo-vascularization, activation of the epithelial-to-mesenchymal transition (EMT), evasion of tumor-targeting immune response. Inhibitors of developmental pathways may prevent the acquisition of new properties by the cancer cells, such as virus-induced transcriptional, metabolic, and functional reprogramming. Each color is associated with a specific virus and the cell processes controlled the developmental pathway.

TABLE 1 | Inhibitors of developmental pathways as therapeutic strategies in viral carcinogenesis.

\begin{tabular}{|c|c|c|c|c|c|}
\hline $\begin{array}{l}\text { Developmental } \\
\text { pathway }\end{array}$ & Inhibitors & Virus & Disease & Effects & References \\
\hline \multirow[t]{3}{*}{ Hedgehog } & GANT-61 & KSHV & Kaposi's sarcoma & $\begin{array}{l}\text { Reduces tumor-sphere } \\
\text { formation }\end{array}$ & Asha et al., 2020 \\
\hline & & HPV & Cervical cancer & $\begin{array}{l}\text { Decreases proliferation survival and } \\
\text { migration }\end{array}$ & Samarzija and Beard, 2012 \\
\hline & Vismodegib & $\mathrm{HBV}, \mathrm{HCV}$ & Chronic hepatitis, liver cirrhosis & $\begin{array}{l}\text { Decreases liver fibrosis in murine } \\
\text { models }\end{array}$ & Kumar et al., 2019 \\
\hline \multirow[t]{4}{*}{ Notch } & GSIX & $\mathrm{H} 1 \mathrm{~N} 1$ & Influenza & $\begin{array}{l}\text { Decreases IFN } \gamma \text { production, increases } \\
\text { viral load }\end{array}$ & Ito et al., 2011 \\
\hline & LY-411575 & $\mathrm{KSHV}$ & Kaposi's sarcoma & $\begin{array}{l}\text { Induces apoptosis of KSHV-infected } \\
\text { cells }\end{array}$ & Curry et al., 2005 \\
\hline & CompE and DBZ & EBV & EBV-associated lymphomas & $\begin{array}{l}\text { Induce EBV-lytic cycle, leading to cell } \\
\text { death }\end{array}$ & Giunco et al., 2015 \\
\hline & DAPT & $\mathrm{HCV}$ & Chronic hepatitis C & $\begin{array}{l}\text { Increases immunosurveillance by } \\
\text { down-regulating } \\
\text { IL-22, IL-17, Tregs }\end{array}$ & $\begin{array}{l}\text { Jiang B.C. et al., 2017; Qin } \\
\text { et al., } 2017\end{array}$ \\
\hline \multirow[t]{2}{*}{ Wnt } & ICG-001 & EBV & NPC & $\begin{array}{l}\text { Down-regulates CD44 via } \beta \text {-catenin } \\
\text { in vitro }\end{array}$ & Chan et al., 2019 \\
\hline & PRI-724 & $\mathrm{HCV}$ & HCV-associated fibrosis & $\begin{array}{l}\text { Decreases liver fibrosis in humans liver } \\
\text { cirrhosis }\end{array}$ & $\begin{array}{l}\text { Kimura et al., 2017; Tokunaga } \\
\text { et al., } 2017\end{array}$ \\
\hline
\end{tabular}

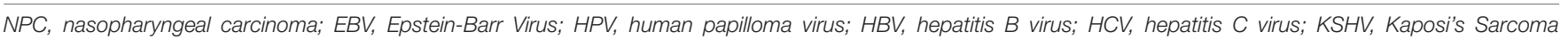

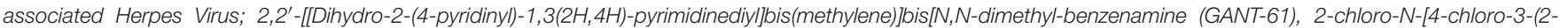

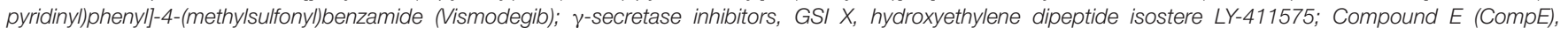

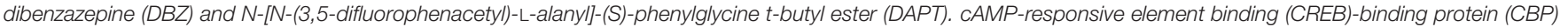
inhibitor (ICG-001) and Potent, specific Wnt pathway inhibitor (PRI-724).

Collectively, the data suggest that dysregulating WNT signaling is an effective mechanism by which oncogenic viruses target and manipulate multiple normal cellular processes to facilitate tumorigenesis. Furthermore, these viruses often employ multiple means of circumventing normal WNT signaling. HBV, HCV, EBV, HPV, and KSHV can all inhibit various components of the WNT pathway that normally keep it in its "OFF" state. These same viruses can also enhance positive regulators of WNT 
signaling, resulting in increased expression of WNT target genes. Thus, by "releasing the brakes and stepping on the gas" of the WNT signaling pathway, oncogenic viruses have evolved to be very efficient at driving cell proliferation, survival, and ultimately, transformation.

\section{HH, NOTCH AND WNT PATHWAYS: INTERSECTION FOR COMMON THERAPEUTIC TARGETS}

Given the complexity of the crosstalk between viruses and the multiple members of the developmental pathways, it is of paramount importance to better understand the molecular mechanisms involved in host-pathogen interactions to develop novel therapies. Notwithstanding the multitude of signaling proteins participating in each developmental pathway, the biological outcome is the same, thus leading to proliferation, cell survival, angiogenesis, stemness, EMT, immune evasion, and maintenance of latent viral state. The ability of the same viral protein to connect cellular proteins of the different pathways, such as HPV E6 or E7 with members of the Notch, $\mathrm{HH}$, and WNT pathways raises the question whether simultaneous or ordered interactions occur in enhanced cell proliferation or tumor progression. Conversely, different viral proteins can interact with signaling proteins of the same pathway. For instance, $\mathrm{HBx}$ and $\mathrm{NS} 3 / 5 \mathrm{~A} / 5 \mathrm{~B}$ encoded by $\mathrm{HBV}$ and $\mathrm{HCV}$, respectively, interact with the same $\mathrm{HH}$ signaling proteins, leading to cell proliferation and survival. GLI1/2 is activated by $\mathrm{HBV}, \mathrm{HPV}, \mathrm{HCV}, \mathrm{KSHV}$, and $\mathrm{EBV}$ proteins bringing to cell proliferation and survival, as well as virus immune evasion (Figure 5). Oncogenic JAG1 signaling is induced by $\mathrm{HBx}$, Tax and LANA. The commonly targeted $\beta$-catenin by HPV and $\mathrm{HCV}$ induces stemness, proliferation and survival in the host cell (Figure 5). Therefore, viral proteins such as $\mathrm{HBx}$, E6E7, Tax, NS3/5A/5B, LANA, EBNA1/2, and LMP1 could be targeted to block viral communication with the components of each developmental pathway (Figure 5). On the other hand, already available inhibitors of each pathway might be used as therapeutic strategies for virus-associated diseases (Table 1). For instance, GANT-61 inhibited HH pathway members, GLI1 and GLI2 in HPV and KSHV associated tumors (Samarzija and Beard, 2012; Asha et al., 2020). Vismodegib, a SMO inhibitor that effectively terminates $\mathrm{HH}$ signaling, decreased liver fibrosis induced by HBV and HCV infection (Kumar et al., 2019). GSI repressed Notch signaling induced by KSHV, EBV, and HCV in associated diseases (Ito et al., 2011; Giunco et al., 2015; Jiang B.C. et al., 2017; Qin et al., 2017). Inhibitors of the WNT pathway decreased oncogenic signaling in EBV and HCV associated diseases (Kimura et al., 2017; Tokunaga et al., 2017; Chan et al., 2019). RIN1 could be used to inhibit RBP-Jk, a Notch pathway molecule exploited by HTLV-1-encoding Tax and EBV-encoding EBNA2, or antibodies against JAG1 might be used to inhibit the oncogenic action of HBV-encoding HBx, HTLV-1-encoding Tax and KSHV-encoding LANA. Similarly, inhibitors against GLI1 and -2 could be used against HBV, HPV, HCV and KSHV associated malignancies and the $\beta$-catenin inhibitor (ICG-001), might be used to target HPV and HCV proteins (Table 1 and Figure 5).

In addition, developmental pathways may interact with each other to form a complex intertwined network connected by common molecules. Thus, understanding the crosstalk between developmental pathways might help to reveal common, druggable targets. This could be the case with $\beta$-catenin, a transcriptional activator of the WNT pathway, which also regulates the Notch-regulated transcriptional repressor, Hes1 (Borggrefe et al., 2016). Furthermore, there is a direct interaction between Notch 1 and $\beta$-catenin, the latter having a protective role on Notch1 by reducing its ubiquitination and ultimately activating Hes 1 expression. In addition, during in vitro angiogenesis, the protein complex, NICD/RBP-Jk/ $\beta$-catenin, was formed and directed the differentiation of vascular progenitor cells toward arterial endothelial cells (Yamamizu et al., 2010). In carcinogenesis, WNT signaling activates Notch1 and Notch3 through its ligand, JAG1, in colorectal and OC, respectively (Borggrefe et al., 2016). Crosstalk exists also between $\mathrm{HH}$ and Notch pathways. For instance, GLI2 and JAG1 induced expression of each other in OC cells (Steg et al., 2011). Although most studies showed a positive feedback loop between components of the developmental pathways, we cannot exclude cases of negative feedback, especially when designing drugs that target specific common molecules.

Considering the complexity of signaling cascades in each developmental pathway and the existing crosstalk between them, there is a need to carefully design appropriate targeted therapies to avoid adverse, toxic, or off-target drug effects in patients. It will be mandatory to test these drugs in appropriate cellular models in vitro, including combined with three-dimensional cell models, which better recapitulate the TME, as well as in vivo experiments using robust animal models to fully evaluate how these drugs might influence anti-viral/anti-tumor immune responses in the host.

\section{FUTURE PERSPECTIVES}

Developmental pathways are important mediators of the transformation potential of different oncogenic viruses. They can turn normal physiologic pathways into potent carcinogenic routes, either to promote aberrant proliferation and acquisition of stemness, and/or to evade immune surveillance. Future efforts focused on better dissecting the cross-signaling between cellular developmental pathways and viruses will further our understanding of the evolution of viral carcinogenesis, including host-pathogen communications that can also shape the cells in the tumor microenvironment and modulate anti-tumoral immune responses. Given the ability of viruses to behave as forced activators of developmental pathways, we should consider targeting their members in future experimental studies. Viruses can repress signaling pathways at early stages of carcinogenesis, as HPV does with TGF $\beta$ and Notch, but can promote later activation, as seen in malignant progression (Meyers et al., 2018). Moreover, as suggested by experimental works in HTLV-1-infected cells and human ATL, inhibitors 
of developmental pathways may represent drug candidates for intractable human diseases (Takahashi et al., 2014). Taken together, we propose that developmental pathway inhibitors have the potential to attenuate tumor development and further research would underpin their role in combined cancer immunotherapy.

\section{AUTHOR CONTRIBUTIONS}

SKP and EM researched the literature and performed the literature review and helped in editing the figures. CM, DB, $\mathrm{SC}$, and RP critically revised the manuscript. PT participated to wrote and to edit the manuscript and figures. EA, LM, and MPF put forward the idea of the manuscript, wrote and edited the

\section{REFERENCES}

Aicher, S., Kakkanas, A., Cohen, L., Blumen, B., Oprisan, G., Njouom, R., et al. (2018). Differential regulation of the $\mathrm{Wnt} / \beta$-catenin pathway by hepatitis $\mathrm{C}$ virus recombinants expressing core from various genotypes. Sci. Rep. 8:11185. doi: 10.1038/s41598-018-29078-2

Al-Shabanah, O. A., Hafez, M. M., Hassan, Z. K., Sayed-Ahmed, M. M., Abozeed, W. N., Alsheikh, A., et al. (2014). Methylation of SFRPs and APC genes in ovarian cancer infected with high risk human papillomavirus. Asian Pac. J. Cancer Prev. 15, 2719-2725. doi: 10.7314/apjcp.2014.15.6.2719

Anastasiadou, E., Stroopinsky, D., Alimperti, S., Jiao, A. L., Pyzer, A. R., Cippitelli, C., et al. (2019). Epstein-Barr virus-encoded EBNA2 alters immune checkpoint PD-L1 expression by downregulating miR-34a in B-cell lymphomas. Leukemia 33, 132-147. doi: 10.1038/s41375-018-0178-x

Andersen, P., Uosaki, H., Shenje, L. T., and Kwon, C. (2012). Non-canonical Notch signaling: emerging role and mechanism. Trends Cell Biol. 22, 257-265. doi: $10.1016 /$ j.tcb.2012.02.003

Arensdorf, A. M., Marada, S., and Ogden, S. K. (2016). Smoothened regulation: a tale of two signals. Trends Pharmacol. Sci. 37, 62-72. doi: 10.1016/j.tips.2015.09. 001

Artavanis-Tsakonas, S., Rand, M. D., and Lake, R. J. (1999). Notch signaling: cell fate control and signal integration in development. Science 284, 770-776. doi: $10.1126 /$ science.284.5415.770

Arzumanyan, A., Friedman, T., Ng, I. O., Clayton, M. M., Lian, Z., and Feitelson, M. A. (2011). Does the hepatitis B antigen HBx promote the appearance of liver cancer stem cells? Cancer Res. 71, 3701-3708. doi: 10.1158/0008-5472.Can-103951

Arzumanyan, A., Sambandam, V., Clayton, M. M., Choi, S. S., Xie, G., Diehl, A. M., et al. (2012). Hedgehog signaling blockade delays hepatocarcinogenesis induced by hepatitis B virus X protein. Cancer Res. 72, 5912-5920. doi: 10.1158/00085472.Can-12-2329

Asha, K., Balfe, N., and Sharma-Walia, N. (2020). Concurrent control of the Kaposi's sarcoma-associated herpesvirus life cycle through chromatin modulation and host hedgehog signaling: a new prospect for the therapeutic potential of lipoxin A4. J. Virol. 94:e02177-19. doi: 10.1128/jvi.021 77-19

Aster, J. C., Pear, W. S., and Blacklow, S. C. (2017). The varied roles of notch in cancer. Annu. Rev. Pathol. 12, 245-275. doi: 10.1146/annurev-pathol-052016100127

Ayaz, F., and Osborne, B. A. (2014). Non-canonical notch signaling in cancer and immunity. Front. Oncol. 4:345. doi: 10.3389/fonc.2014.00345

Bartoszewski, R., Brewer, J. W., Rab, A., Crossman, D. K., Bartoszewska, S., Kapoor, N., et al. (2011). The unfolded protein response (UPR)-activated transcription factor X-box-binding protein 1 (XBP1) induces microRNA-346 expression that targets the human antigen peptide transporter 1 (TAP1) mRNA and governs immune regulatory genes. J. Biol. Chem. 286, 41862-41870. doi: 10.1074/jbc. M111.304956 manuscript, figures, and the table. All authors contributed to the article and approved the submitted version.

\section{FUNDING}

This work was supported by LILT, Italy, to PT and Ateneo (RP11916888BC2710) 2019 to MPF.

\section{ACKNOWLEDGMENTS}

This article is dedicated to the memory of Alberto Gulino. The authors thank Martina Del Gaizo and Ilaria Sergio for their valuable help in conceptualizing and creating figures.

Bellon, M., Moles, R., Chaib-Mezrag, H., Pancewicz, J., and Nicot, C. (2018). JAG1 overexpression contributes to Notch1 signaling and the migration of HTLV1-transformed ATL cells. J. Hematol. Oncol. 11:119. doi: 10.1186/s13045-0180665-6

Bernasconi-Elias, P., Hu, T., Jenkins, D., Firestone, B., Gans, S., Kurth, E., et al. (2016). Characterization of activating mutations of NOTCH3 in T-cell acute lymphoblastic leukemia and anti-leukemic activity of NOTCH3 inhibitory antibodies. Oncogene 35, 6077-6086. doi: 10.1038/onc.2016.133

Birdwell, C. E., Prasai, K., Dykes, S., Jia, Y., Munroe, T. G. C., Bienkowska-Haba, M., et al. (2018). Epstein-Barr virus stably confers an invasive phenotype to epithelial cells through reprogramming of the WNT pathway. Oncotarget 9 , 10417-10435. doi: 10.18632/oncotarget.23824

Birdwell, C. E., Queen, K. J., Kilgore, P. C., Rollyson, P., Trutschl, M., Cvek, U., et al. (2014). Genome-wide DNA methylation as an epigenetic consequence of Epstein-Barr virus infection of immortalized keratinocytes. J. Virol. 88, 11442-11458. doi: 10.1128/jvi.00972-14

Borggrefe, T., and Oswald, F. (2009). The Notch signaling pathway: transcriptional regulation at Notch target genes. Cell. Mol. Life Sci. 66, 1631-1646. doi: 10.1007/ s00018-009-8668-7

Borggrefe, T., Lauth, M., Zwijsen, A., Huylebroeck, D., Oswald, F., and Giaimo, B. D. (2016). The Notch intracellular domain integrates signals from Wnt, Hedgehog, TGFß/BMP and hypoxia pathways. Biochim. Biophys. Acta 1863, 303-313. doi: 10.1016/j.bbamcr.2015.11.020

Carpenter, R. L., and Lo, H. W. (2012). Hedgehog pathway and GLI1 isoforms in human cancer. Discov. Med. 13, 105-113.

Chan, L. S., Man, O. Y., Kwok, H. H., Chen, L., Chan, K. C., Lung, H. L., et al. (2019). The Wnt modulator ICG-001 mediates the inhibition of nasopharyngeal carcinoma cell migration in vitro via the miR-150/CD44 axis. Int. J. Oncol. 54, 1010-1020. doi: 10.3892/ijo.2018.4664

Chandiran, K., Lawlor, R., Pannuti, A., Perez, G. G., Srinivasan, J., Golde, T. E., et al. (2018). Notch1 primes CD4 T cells for T helper type I differentiation through its early effects on miR-29. Mol. Immunol. 99, 191-198. doi: 10.1016/j.molimm. 2018.05.002

Chen, P. M., Cheng, Y. W., Wang, Y. C., Wu, T. C., Chen, C. Y., and Lee, H. (2014). Up-regulation of FOXM1 by E6 oncoprotein through the MZF1/NKX2-1 axis is required for human papillomavirus-associated tumorigenesis. Neoplasia 16, 961-971. doi: 10.1016/j.neo.2014.09.010

Chen, Q., Cao, H. Z., and Zheng, P. S. (2014). LGR5 promotes the proliferation and tumor formation of cervical cancer cells through the Wnt/ $\beta$-catenin signaling pathway. Oncotarget 5, 9092-9105. doi: 10.18632/oncotarget.2377

Chen, X., Lingala, S., Khoobyari, S., Nolta, J., Zern, M. A., and Wu, J. (2011). Epithelial mesenchymal transition and hedgehog signaling activation are associated with chemoresistance and invasion of hepatoma subpopulations. J. Hepatol. 55, 838-845. doi: 10.1016/j.jhep.2010.12.043

Chen, Z., Tang, J., Cai, X., Huang, Y., Gao, Q., Liang, L., et al. (2016). HBx mutations promote hepatoma cell migration through the $\mathrm{Wnt} / \beta$-catenin signaling pathway. Cancer Sci. 107, 1380-1389. doi: 10.1111/cas.13014 
Cheng, W., Zheng, T., Wang, Y., Cai, K., Wu, W., Zhao, T., et al. (2019). Activation of Notch1 signaling by HTLV-1 Tax promotes proliferation of adult T-cell leukemia cells. Biochem. Biophys. Res. Commun. 512, 598-603. doi: 10.1016/j. bbrc.2019.03.094

Chiara, M., Manzari, C., Lionetti, C., Mechelli, R., Anastasiadou, E., Chiara Buscarinu, M., et al. (2016). Geographic population structure in epstein-barr virus revealed by comparative genomics. Genome Biol. Evol. 8, 3284-3291. doi: $10.1093 /$ gbe/evw226

Chiorean, E. G., LoRusso, P., Strother, R. M., Diamond, J. R., Younger, A., Messersmith, W. A., et al. (2015). A phase I first-in-human study of enoticumab (REGN421), a fully human delta-like ligand 4 (Dll4) monoclonal antibody in patients with advanced solid tumors. Clin. Cancer Res. 21, 2695-2703. doi: 10.1158/1078-0432.Ccr-14-2797

Chou, H. H., Chien, W. H., Wu, L. L., Cheng, C. H., Chung, C. H., Horng, J. H., et al. (2015). Age-related immune clearance of hepatitis B virus infection requires the establishment of gut microbiota. Proc. Natl. Acad. Sci. U.S.A. 112, 2175-2180. doi: 10.1073/pnas.1424775112

Colditz, J., Thiele, S., Baschant, U., Garbe, A. I., Niehrs, C., Hofbauer, L. C., et al. (2019). Osteogenic Dkk1 mediates glucocorticoid-induced but not arthritisinduced bone loss. J. Bone Miner. Res. 34, 1314-1323. doi: 10.1002/jbmr.3702

Colombo, M., Mirandola, L., Chiriva-Internati, M., Basile, A., Locati, M., Lesma, E., et al. (2018). Cancer cells exploit notch signaling to redefine a supportive cytokine milieu. Front. Immunol. 9:1823. doi: 10.3389/fimmu.2018.01823

Crompton, T., Outram, S. V., and Hager-Theodorides, A. L. (2007). Sonic hedgehog signalling in T-cell development and activation. Nat. Rev. Immunol. 7, 726-735. doi: 10.1038/nri2151

Curry, C. L., Reed, L. L., Golde, T. E., Miele, L., Nickoloff, B. J., and Foreman, K. E. (2005). Gamma secretase inhibitor blocks Notch activation and induces apoptosis in Kaposi's sarcoma tumor cells. Oncogene 24, 6333-6344. doi: 10. 1038/sj.onc. 1208783

Deb Pal, A., and Banerjee, S. (2015). Epstein-Barr virus latent membrane protein 2A mediated activation of Sonic Hedgehog pathway induces HLA class Ia downregulation in gastric cancer cells. Virology 484, 22-32. doi: 10.1016/j.virol. 2015.05.007

D’Souza, B., Miyamoto, A., and Weinmaster, G. (2008). The many facets of Notch ligands. Oncogene 27, 5148-5167. doi: 10.1038/onc.2008.229

Dubuisson, J., and Cosset, F. L. (2014). Virology and cell biology of the hepatitis C virus life cycle: an update. J. Hepatol. 61 (Suppl. 1), S3-S13. doi: 10.1016/j.jhep. 2014.06.031

Eldeeb, M. K., Magour, G. M., Bedair, R. N., Shamseya, M. M., and Hammouda, M. A. (2020). Study of Dickkopf-1 (DKK-1) in patients with chronic viral hepatitis C-related liver cirrhosis with and without hepatocellular carcinoma. Clin. Exp. Hepatol. 6, 85-91. doi: 10.5114/ceh.2020.95831

Elmore, L. W., Hancock, A. R., Chang, S. F., Wang, X. W., Chang, S., Callahan, C. P., et al. (1997). Hepatitis B virus X protein and p53 tumor suppressor interactions in the modulation of apoptosis. Proc. Natl. Acad. Sci. U.S.A. 94, 14707-14712. doi: 10.1073/pnas.94.26.14707

Esser, A. K., Rauch, D. A., Xiang, J., Harding, J. C., Kohart, N. A., Ross, M. H., et al. (2017). HTLV-1 viral oncogene HBZ induces osteolytic bone disease in transgenic mice. Oncotarget 8, 69250-69263. doi: 10.18632/oncotarget.20565

Feitelson, M. A., Zhu, M., Duan, L. X., and London, W. T. (1993). Hepatitis B x antigen and p53 are associated in vitro and in liver tissues from patients with primary hepatocellular carcinoma. Oncogene 8, 1109-1117.

Ferrandino, F., Bernardini, G., Tsaouli, G., Grazioli, P., Campese, A. F., Noce, C., et al. (2018a). Intrathymic Notch3 and CXCR4 combinatorial interplay facilitates T-cell leukemia propagation. Oncogene 37, 6285-6298. doi: 10.1038/ s41388-018-0401-2

Ferrandino, F., Grazioli, P., Bellavia, D., Campese, A. F., Screpanti, I., and Felli, M. P. (2018b). Notch and NF- $\kappa$ B: coach and players of regulatory T-Cell response in cancer. Front. Immunol. 9:2165. doi: 10.3389/fimmu.2018.02165

Ferrando, A. A. (2009). The role of NOTCH1 signaling in T-ALL. Hematol. Am. Soc. Hematol. Educ. Program 2009, 353-361. doi: 10.1182/asheducation-2009.1. 353

Forte, E., and Luftig, M. A. (2011). The role of microRNAs in Epstein-Barr virus latency and lytic reactivation. Microbes Infect. 13, 1156-1167. doi: 10.1016/j. micinf.2011.07.007

Fragoso-Ontiveros, V., María Alvarez-García, R., Contreras-Paredes, A., VacaPaniagua, F., Alonso Herrera, L., López-Camarillo, C., et al. (2012). Gene expression profiles induced by E6 from non-European HPV18 variants reveals a differential activation on cellular processes driving to carcinogenesis. Virology 432, 81-90. doi: 10.1016/j.virol.2012.05.029

Fujimuro, M., and Hayward, S. D. (2003). The latency-associated nuclear antigen of Kaposi's sarcoma-associated herpesvirus manipulates the activity of glycogen synthase kinase-3beta. J. Virol. 77, 8019-8030. doi: 10.1128/jvi.77.14.8019-8030. 2003

Fujimuro, M., Wu, F. Y., ApRhys, C., Kajumbula, H., Young, D. B., Hayward, G. S., et al. (2003). A novel viral mechanism for dysregulation of beta-catenin in Kaposi's sarcoma-associated herpesvirus latency. Nat. Med. 9, 300-306. doi: 10.1038/nm829

Fujita, K., and Janz, S. (2007). Attenuation of WNT signaling by DKK-1 and 2 regulates $\mathrm{BMP} 2$-induced osteoblast differentiation and expression of OPG, RANKL and M-CSF. Mol. Cancer 6:71. doi: 10.1186/1476-4598-6-71

Gaglia, M. M., and Munger, K. (2018). More than just oncogenes: mechanisms of tumorigenesis by human viruses. Curr. Opin. Virol. 32, 48-59. doi: 10.1016/j. coviro.2018.09.003

Gao, J., Chen, C., Hong, L., Wang, J., Du, Y., Song, J., et al. (2007). Expression of Jagged 1 and its association with hepatitis B virus $\mathrm{X}$ protein in hepatocellular carcinoma. Biochem. Biophys. Res. Commun. 356, 341-347. doi: 10.1016/j.bbrc. 2007.02.130

Gao, J., Xiong, Y., Wang, Y., Wang, Y., Zheng, G., and Xu, H. (2016). Hepatitis B virus $\mathrm{X}$ protein activates Notch signaling by its effects on Notch 1 and Notch 4 in human hepatocellular carcinoma. Int. J. Oncol. 48, 329-337. doi: 10.3892/ijo. 2015.3221

Giunco, S., Celeghin, A., Gianesin, K., Dolcetti, R., Indraccolo, S., and De Rossi, A. (2015). Cross talk between EBV and telomerase: the role of TERT and NOTCH2 in the switch of latent/lytic cycle of the virus. Cell Death Dis. 6:e1774. doi: $10.1038 /$ cddis.2015.145

Gordon, W. R., Arnett, K. L., and Blacklow, S. C. (2008). The molecular logic of Notch signaling-a structural and biochemical perspective. J. Cell Sci. 121(Pt 19), 3109-3119. doi: 10.1242/jcs.035683

Grazioli, P., Felli, M. P., Screpanti, I., and Campese, A. F. (2017). The mazy case of Notch and immunoregulatory cells. J. Leukoc. Biol. 102, 361-368. doi: 10.1189/jlb.1VMR1216-505R

Harms, P. W., Harms, K. L., Moore, P. S., DeCaprio, J. A., Nghiem, P., Wong, M. K. K., et al. (2018). The biology and treatment of Merkel cell carcinoma: current understanding and research priorities. Nat. Rev. Clin. Oncol. 15, 763776. doi: 10.1038/s41571-018-0103-2

Hayward, S. D. (2004). Viral interactions with the Notch pathway. Semin. Cancer Biol. 14, 387-396. doi: 10.1016/j.semcancer.2004 .04 .018

Höfelmayr, H., Strobl, L. J., Marschall, G., Bornkamm, G. W., and Zimber-Strobl, U. (2001). Activated Notch1 can transiently substitute for EBNA2 in the maintenance of proliferation of LMP1-expressing immortalized B cells. J. Virol. 75, 2033-2040. doi: 10.1128/jvi.75.5.2033-2040.2001

Hurtado, C., Safarova, A., Smith, M., Chung, R., Bruyneel, A. A. N., GomezGaleno, J., et al. (2019). Disruption of NOTCH signaling by a small molecule inhibitor of the transcription factor RBPJ. Sci. Rep. 9:10811. doi: 10.1038/ s41598-019-46948-5

Hyun, J., and Jung, Y. (2016). MicroRNAs in liver fibrosis: focusing on the interaction with hedgehog signaling. World J. Gastroenterol. 22, 6652-6662. doi: 10.3748/wjg.v22.i29.6652

Iriana, S., Asha, K., Repak, M., and Sharma-Walia, N. (2021). Hedgehog signaling: implications in cancers and viral infections. Int. J. Mol. Sci. 22:1042. doi: 10. 3390/ijms22031042

Ishida, T., Ishii, T., Inagaki, A., Yano, H., Komatsu, H., Iida, S., et al. (2006). Specific recruitment of CC chemokine receptor 4-positive regulatory $\mathrm{T}$ cells in Hodgkin lymphoma fosters immune privilege. Cancer Res. 66, 5716-5722. doi: 10.1158/0008-5472.Can-06-0261

Ito, T., Allen, R. M., Carson, W. F. T., Schaller, M., Cavassani, K. A., Hogaboam, C. M., et al. (2011). The critical role of Notch ligand Delta-like 1 in the pathogenesis of influenza A virus (H1N1) infection. PLoS Pathog. 7:e1002341. doi: 10.1371/journal.ppat.1002341

Iwai, A., Takegami, T., Shiozaki, T., and Miyazaki, T. (2011). Hepatitis C virus NS3 protein can activate the Notch-signaling pathway through binding to a transcription factor, SRCAP. PLoS One 6:e20718. doi: 10.1371/journal.pone. 0020718 
Javanmard, D., Najafi, M., Babaei, M. R., Karbalaie Niya, M. H., Esghaei, M., Panahi, M., et al. (2020). Investigation of CTNNB1 gene mutations and expression in hepatocellular carcinoma and cirrhosis in association with hepatitis B virus infection. Infect. Agent Cancer 15:37. doi: 10.1186/s13027-02000297-5

Jiang, B. C., Liu, X., Liu, X. H., Li, Z. S., and Zhu, G. Z. (2017). Notch signaling regulates circulating $T$ helper 22 cells in patients with chronic hepatitis C. Viral Immunol. 30, 522-532. doi: 10.1089/vim.2017.0007

Jiang, X. H., Xie, Y. T., Cai, Y. P., Ren, J., and Ma, T. (2017). Effects of hepatitis C virus core protein and nonstructural protein $4 \mathrm{~B}$ on the $\mathrm{Wnt} / \beta$-catenin pathway. BMC Microbiol. 17:124. doi: 10.1186/s12866-017-1032-4

Jundt, F., Anagnostopoulos, I., Förster, R., Mathas, S., Stein, H., and Dörken, B. (2002). Activated Notch1 signaling promotes tumor cell proliferation and survival in Hodgkin and anaplastic large cell lymphoma. Blood 99, 3398-3403. doi: 10.1182/blood.v99.9.3398

Kalluri, R., and Weinberg, R. A. (2009). The basics of epithelial-mesenchymal transition. J. Clin. Invest. 119, 1420-1428. doi: 10.1172/jci39104

Kang, J., Kim, E., Kim, W., Seong, K. M., Youn, H., Kim, J. W., et al. (2013). Rhamnetin and cirsiliol induce radiosensitization and inhibition of epithelialmesenchymal transition (EMT) by miR-34a-mediated suppression of Notch-1 expression in non-small cell lung cancer cell lines. J. Biol. Chem. 288, 2734327357. doi: 10.1074/jbc.M113.490482

Katoh, M. (2007). Networking of WNT, FGF, Notch, BMP, and Hedgehog signaling pathways during carcinogenesis. Stem Cell Rev. 3, 30-38. doi: 10.1007/s12015007-0006-6

Kessler, M., Hoffmann, K., Brinkmann, V., Thieck, O., Jackisch, S., Toelle, B., et al. (2015). The Notch and Wnt pathways regulate stemness and differentiation in human fallopian tube organoids. Nat. Commun. 6:8989. doi: 10.1038/ ncomms 9989

Kim, H. Y., Cho, H. K., Hong, S. P., and Cheong, J. (2011). Hepatitis B virus X protein stimulates the Hedgehog-Gli activation through protein stabilization and nuclear localization of Gli1 in liver cancer cells. Cancer Lett. 309, 176-184. doi: 10.1016/j.canlet.2011.05.033

Kimura, K., Ikoma, A., Shibakawa, M., Shimoda, S., Harada, K., Saio, M., et al. (2017). Safety, tolerability, and preliminary efficacy of the anti-fibrotic small molecule PRI-724, a CBP/ $\beta$-Catenin inhibitor, in patients with hepatitis $\mathrm{C}$ virus-related cirrhosis: a single-center, open-label, dose escalation phase 1 trial. EBioMedicine 23, 79-87. doi: 10.1016/j.ebiom.2017.08.016

Komiya, Y., and Habas, R. (2008). Wnt signal transduction pathways. Organogenesis 4, 68-75. doi: 10.4161/org.4.2.5851

Kong, F., Hu, W., Zhou, K., Wei, X., Kou, Y., You, H., et al. (2016). Hepatitis $B$ virus $X$ protein promotes interleukin-7 receptor expression via NF- $\mathrm{KB}$ and Notch1 pathway to facilitate proliferation and migration of hepatitis B virusrelated hepatoma cells. J. Exp. Clin. Cancer Res. 35:172. doi: 10.1186/s13046016-0448-2

Kongkavitoon, P., Tangkijvanich, P., Hirankarn, N., and Palaga, T. (2016). Hepatitis B virus HBx activates notch signaling via delta-like 4/Notch1 in hepatocellular carcinoma. PLoS One 11:e0146696. doi: 10.1371/journal.pone. 0146696

Kopan, R. (2012). Notch signaling. Cold Spring Harb. Perspect. Biol. 4:a011213. doi: 10.1101/cshperspect.a011213

Kopan, R., and Ilagan, M. X. (2009). The canonical Notch signaling pathway: unfolding the activation mechanism. Cell 137, 216-233. doi: 10.1016/j.cell.2009. 03.045

Krump, N. A., and You, J. (2018). Molecular mechanisms of viral oncogenesis in humans. Nat. Rev. Microbiol. 16, 684-698. doi: 10.1038/s41579-018-0064-6

Kumar, V., Dong, Y., Kumar, V., Almawash, S., and Mahato, R. I. (2019). The use of micelles to deliver potential hedgehog pathway inhibitor for the treatment of liver fibrosis. Theranostics 9, 7537-7555. doi: 10.7150/thno.38913

Kuromi, T., Matsushita, M., Iwasaki, T., Nonaka, D., Kuwamoto, S., Nagata, K., et al. (2017). Association of expression of the hedgehog signal with Merkel cell polyomavirus infection and prognosis of Merkel cell carcinoma. Hum. Pathol. 69, 8-14. doi: 10.1016/j.humpath.2017.05.011

Lan, K., Choudhuri, T., Murakami, M., Kuppers, D. A., and Robertson, E. S. (2006). Intracellular activated Notch1 is critical for proliferation of Kaposi's sarcomaassociated herpesvirus-associated B-lymphoma cell lines in vitro. J. Virol. 80, 6411-6419. doi: 10.1128/jvi.00239-06
Lan, K., Verma, S. C., Murakami, M., Bajaj, B., Kaul, R., and Robertson, E. S. (2007). Kaposi's sarcoma herpesvirus-encoded latency-associated nuclear antigen stabilizes intracellular activated Notch by targeting the Sel10 protein. Proc. Natl. Acad. Sci. U.S.A. 104, 16287-16292. doi: 10.1073/pnas.0703508104

Lathion, S., Schaper, J., Beard, P., and Raj, K. (2003). Notch1 can contribute to viral-induced transformation of primary human keratinocytes. Cancer Res. 63, 8687-8694.

Li, J., Quan, H., Liu, Q., Si, Z., He, Z., and Qi, H. (2013). Alterations of axis inhibition protein 1 (AXIN1) in hepatitis B virus-related hepatocellular carcinoma and overexpression of AXIN1 induces apoptosis in hepatocellular cancer cells. Oncol. Res. 20, 281-288. doi: 10.3727/096504013x13639794277608

Li, S., Hu, H., He, Z., Liang, D., Sun, R., and Lan, K. (2016). Fine-Tuning of the Kaposi's sarcoma-associated herpesvirus life cycle in neighboring cells through the RTA-JAG1-Notch pathway. PLoS Pathog. 12:e1005900. doi: 10.1371/journal. ppat. 1005900

Li, Y., He, X., Zhang, X., Xu, Y., Wu, Y., and Xu, X. (2021). Immunerelated microRNA signature for predicting prognosis and the immune microenvironment in hepatocellular carcinoma. Life Sci. 265:118799. doi: 10. $1016 /$ j.lfs.2020.118799

Liang, T. J. (2009). Hepatitis B: the virus and disease. Hepatology 49 (Suppl. 5), S13-S21. doi: 10.1002/hep.22881

Lichtig, H., Gilboa, D. A., Jackman, A., Gonen, P., Levav-Cohen, Y., Haupt, Y., et al. (2010). HPV16 E6 augments Wnt signaling in an E6AP-dependent manner. Virology 396, 47-58. doi: 10.1016/j.virol.2009.10.011

Logeat, F., Bessia, C., Brou, C., LeBail, O., Jarriault, S., Seidah, N. G., et al. (1998). The Notch1 receptor is cleaved constitutively by a furin-like convertase. Proc. Natl. Acad. Sci. U.S.A. 95, 8108-8112. doi: 10.1073/pnas.95.14.8108

López-Arribillaga, E., Rodilla, V., Colomer, C., Vert, A., Shelton, A., Cheng, J. H., et al. (2018). Manic Fringe deficiency imposes Jagged1 addiction to intestinal tumor cells. Nat. Commun. 9:2992. doi: 10.1038/s41467-018-05385-0

Lum, L., and Beachy, P. A. (2004). The Hedgehog response network: sensors, switches, and routers. Science 304, 1755-1759. doi: 10.1126/science.1098020

Ma, G., Yasunaga, J., Akari, H., and Matsuoka, M. (2015). TCF1 and LEF1 act as T-cell intrinsic HTLV-1 antagonists by targeting Tax. Proc. Natl. Acad. Sci. U.S.A. 112, 2216-2221. doi: 10.1073/pnas.1419198112

Ma, G., Yasunaga, J., Fan, J., Yanagawa, S., and Matsuoka, M. (2013). HTLV-1 bZIP factor dysregulates the Wnt pathways to support proliferation and migration of adult T-cell leukemia cells. Oncogene 32, 4222-4230. doi: 10.1038/onc.2012.450

Mahmoudvand, S., Shokri, S., Taherkhani, R., and Farshadpour, F. (2019). Hepatitis $\mathrm{C}$ virus core protein modulates several signaling pathways involved in hepatocellular carcinoma. World J. Gastroenterol. 25, 42-58. doi: 10.3748/ wjg.v25.i1.42

Mei, S., and Zhang, K. (2016). Computational discovery of Epstein-Barr virus targeted human genes and signalling pathways. Sci. Rep. 6:30612. doi: 10.1038/ srep30612

Meisel, C. T., Porcheri, C., and Mitsiadis, T. A. (2020). Cancer stem cells, quo Vadis? The notch signaling pathway in tumor initiation and progression. Cells 9:1879. doi: 10.3390/cells9081879

Merle, P., de la Monte, S., Kim, M., Herrmann, M., Tanaka, S., Von Dem Bussche, A., et al. (2004). Functional consequences of frizzled-7 receptor overexpression in human hepatocellular carcinoma. Gastroenterology 127, 1110-1122. doi: 10. 1053/j.gastro.2004.07.009

Mesri, E. A., Feitelson, M. A., and Munger, K. (2014). Human viral oncogenesis: a cancer hallmarks analysis. Cell Host Microbe 15, 266-282. doi: 10.1016/j.chom. 2014.02.011

Meyers, J. M., Grace, M., Uberoi, A., Lambert, P. F., and Munger, K. (2018). Inhibition of TGF- $\beta$ and NOTCH signaling by cutaneous papillomaviruses. Front. Microbiol. 9:389. doi: 10.3389/fmicb.2018.00389

Mo, W., Tong, C., Zhang, Y., and Lu, H. (2015). microRNAs' differential regulations mediate the progress of human papillomavirus (HPV)-induced cervical intraepithelial neoplasia (CIN). BMC Syst. Biol. 9:4. doi: 10.1186/ s12918-015-0145-3

Mondal, P., Kaur, B., Natesh, J., and Meeran, S. M. (2021). The emerging role of miRNA in the perturbation of tumor immune microenvironment in chemoresistance: therapeutic implications. Semin. Cell Dev. Biol. S10849521(21)00074-4. doi: 10.1016/j.semcdb.2021.04.001

Morgan, T. H. (1917). The theory of the gene. Am. Nat. 51, 513-544. 
Moriyama, H., Moriyama, M., Ozawa, T., Tsuruta, D., Iguchi, T., Tamada, S., et al. (2018). Notch signaling enhances stemness by regulating metabolic pathways

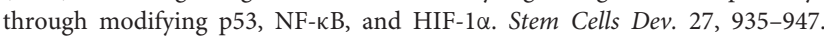
doi: $10.1089 / \mathrm{scd} .2017 .0260$

Niida, A., Hiroko, T., Kasai, M., Furukawa, Y., Nakamura, Y., Suzuki, Y., et al. (2004). DKK1, a negative regulator of Wnt signaling, is a target of the betacatenin/TCF pathway. Oncogene 23, 8520-8526. doi: 10.1038/sj.onc.1207892

Oyervides-Muñoz, M. A., Pérez-Maya, A. A., Rodríguez-Gutiérrez, H. F., GómezMacias, G. S., Fajardo-Ramírez, O. R., Treviño, V., et al. (2018). Understanding the HPV integration and its progression to cervical cancer. Infect. Genet. Evol. 61, 134-144. doi: 10.1016/j.meegid.2018.03.003

Palle, K., Mani, C., Tripathi, K., and Athar, M. (2015). Aberrant GLI1 activation in DNA damage response, carcinogenesis and chemoresistance. Cancers (Basel) 7, 2330-2351. doi: 10.3390/cancers7040894

Pandit, T., and Ogden, S. K. (2017). Contributions of noncanonical smoothened signaling during embryonic development. J. Dev. Biol. 5:11. doi: 10.3390/ jdb5040011

Pelullo, M., Nardozza, F., Zema, S., Quaranta, R., Nicoletti, C., Besharat, Z. M., et al. (2019). Kras/ADAM17-dependent Jag1-ICD reverse signaling sustains colorectal cancer progression and chemoresistance. Cancer Res. 79, 5575-5586. doi: 10.1158/0008-5472.Can-19-0145

Penton, A. L., Leonard, L. D., and Spinner, N. B. (2012). Notch signaling in human development and disease. Semin. Cell Dev. Biol. 23, 450-457. doi: 10.1016/j. semcdb.2012.01.010

Pereira Tde, A., Witek, R. P., Syn, W. K., Choi, S. S., Bradrick, S., Karaca, G. F., et al. (2010). Viral factors induce Hedgehog pathway activation in humans with viral hepatitis, cirrhosis, and hepatocellular carcinoma. Lab. Invest. 90, 1690-1703. doi: 10.1038/labinvest.2010.147

Piirsoo, A., Pink, A., Kasak, L., Kala, M., Kasvandik, S., Ustav, M., et al. (2019). Differential phosphorylation determines the repressor and activator potencies of GLI1 proteins and their efficiency in modulating the HPV life cycle. PLoS One 14:e0225775. doi: 10.1371/journal.pone.0225775

Polakowski, N., Gregory, H., Mesnard, J. M., and Lemasson, I. (2010). Expression of a protein involved in bone resorption, Dkk1, is activated by HTLV-1 bZIP factor through its activation domain. Retrovirology 7:61. doi: 10.1186/1742-4690-7-61

Port, R. J., Pinheiro-Maia, S., Hu, C., Arrand, J. R., Wei, W., Young, L. S., et al. (2013). Epstein-Barr virus induction of the Hedgehog signalling pathway imposes a stem cell phenotype on human epithelial cells. J. Pathol. 231, 367-377. doi: $10.1002 /$ path.4245

Prendergast, G. C. (2008). Immune escape as a fundamental trait of cancer: focus on IDO. Oncogene 27, 3889-3900. doi: 10.1038/onc.2008.35

Qi, X., and Li, X. (2020). Mechanistic insights into the generation and transduction of hedgehog signaling. Trends Biochem. Sci. 45, 397-410. doi: 10.1016/j.tibs. 2020.01.006

Qin, L., Zhou, Y. C., Wu, H. J., Zhuo, Y., Wang, Y. P., Si, C. Y., et al. (2017). Notch signaling modulates the balance of regulatory $\mathrm{T}$ cells and $\mathrm{T}$ helper 17 cells in patients with chronic hepatitis C. DNA Cell Biol. 36, 311-320. doi: 10.1089/dna.2016.3609

Quaranta, R., Pelullo, M., Zema, S., Nardozza, F., Checquolo, S., Lauer, D. M., et al. (2017). Maml1 acts cooperatively with Gli proteins to regulate sonic hedgehog signaling pathway. Cell Death Dis. 8:e2942. doi: 10.1038/cddis.2017.326

Rath, G., Jawanjal, P., Salhan, S., Nalliah, M., and Dhawan, I. (2015). Clinical significance of inactivated glycogen synthase kinase $3 \beta$ in HPV-associated cervical cancer: relationship with $\mathrm{Wnt} / \beta$-catenin pathway activation. Am. J. Reprod. Immunol. 73, 460-478. doi: 10.1111/aji.12346

Rodríguez-Sastre, M. A., González-Maya, L., Delgado, R., Lizano, M., Tsubaki, G., Mohar, A., et al. (2005). Abnormal distribution of E-cadherin and beta-catenin in different histologic types of cancer of the uterine cervix. Gynecol. Oncol. 97, 330-336. doi: 10.1016/j.ygyno.2004.12.062

Roo, J. J. D., and Staal, F. J. T. (2020). Cell signaling pathway reporters in adult hematopoietic stem cells. Cells 9:2264. doi: 10.3390/cells9102264

Samarzija, I., and Beard, P. (2012). Hedgehog pathway regulators influence cervical cancer cell proliferation, survival and migration. Biochem. Biophys. Res. Commun. 425, 64-69. doi: 10.1016/j.bbrc.2012.07.051

Sanders, P. G., Muñoz-Descalzo, S., Balayo, T., Wirtz-Peitz, F., Hayward, P., and Arias, A. M. (2009). Ligand-independent traffic of Notch buffers activated Armadillo in Drosophila. PLoS Biol. 7:e1000169. doi: 10.1371/journal.pbio. 1000169
Sharma, A., Paranjape, A. N., Rangarajan, A., and Dighe, R. R. (2012). A monoclonal antibody against human Notch1 ligand-binding domain depletes subpopulation of putative breast cancer stem-like cells. Mol. Cancer Ther. 11, 77-86. doi: 10.1158/1535-7163.Mct-11-0508

Sjölund, J., Manetopoulos, C., Stockhausen, M. T., and Axelson, H. (2005). The Notch pathway in cancer: differentiation gone awry. Eur. J. Cancer 41, 26202629. doi: 10.1016/j.ejca.2005.06.025

Stamos, J. L., and Weis, W. I. (2013). The $\beta$-catenin destruction complex. Cold Spring Harb. Perspect. Biol. 5:a007898. doi: 10.1101/cshperspect.a007898

Steg, A. D., Katre, A. A., Goodman, B., Han, H. D., Nick, A. M., Stone, R. L., et al. (2011). Targeting the notch ligand JAGGED1 in both tumor cells and stroma in ovarian cancer. Clin. Cancer Res. 17, 5674-5685. doi: 10.1158/1078-0432.Ccr11-0432

Strobl, L. J., Höfelmayr, H., Marschall, G., Brielmeier, M., Bornkamm, G. W., and Zimber-Strobl, U. (2000). Activated Notch1 modulates gene expression in B cells similarly to Epstein-Barr viral nuclear antigen 2. J. Virol. 74, 1727-1735. doi: 10.1128/jvi.74.4.1727-1735.2000

Strobl, L. J., Höfelmayr, H., Stein, C., Marschall, G., Brielmeier, M., Laux, G., et al. (1997). Both Epstein-Barr viral nuclear antigen 2 (EBNA2) and activated Notch1 transactivate genes by interacting with the cellular protein RBP-J kappa. Immunobiology 198, 299-306. doi: 10.1016/s0171-2985(97)80050-2

Sun, Q., Wang, R., Luo, J., Wang, P., Xiong, S., Liu, M., et al. (2014). Notch 1 promotes hepatitis B virus X protein-induced hepatocarcinogenesis via Wnt/ß-catenin pathway. Int. J. Oncol. 45, 1638-1648. doi: 10.3892/ijo.2014. 2537

Takahashi, R., Yamagishi, M., Nakano, K., Yamochi, T., Yamochi, T., Fujikawa, D., et al. (2014). Epigenetic deregulation of Ellis Van Creveld confers robust Hedgehog signaling in adult T-cell leukemia. Cancer Sci. 105, 1160-1169. doi: $10.1111 /$ cas. 12480

Takebe, N., Miele, L., Harris, P. J., Jeong, W., Bando, H., Kahn, M., et al. (2015). Targeting Notch, Hedgehog, and Wnt pathways in cancer stem cells: clinical update. Nat. Rev. Clin. Oncol. 12, 445-464. doi: 10.1038/nrclinonc.2015.61

Tang, Z., Chen, W., Xu, Y., Lin, X., Liu, X., Li, Y., et al. (2020). miR-4721, Induced by EBV-miR-BART22, targets GSK3 $\beta$ to enhance the tumorigenic capacity of NPC through the WNT/ $\beta$-catenin Pathway. Mol. Ther. Nucleic Acids 22, 557-571. doi: 10.1016/j.omtn.2020.09.021

Tierney, R. J., Nagra, J., Rowe, M., Bell, A. I., and Rickinson, A. B. (2015). The Epstein-Barr virus BamHI C promoter is not essential for B cell immortalization in vitro, but it greatly enhances B cell growth transformation. J. Virol. 89, 2483-2493. doi: 10.1128/jvi.03300-14

Tokunaga, Y., Osawa, Y., Ohtsuki, T., Hayashi, Y., Yamaji, K., Yamane, D., et al. (2017). Selective inhibitor of $\mathrm{Wnt} / \beta$-catenin/CBP signaling ameliorates hepatitis C virus-induced liver fibrosis in mouse model. Sci. Rep. 7:325. doi: 10.1038/s41598-017-00282-w

Tottone, L., Zhdanovskaya, N., Carmona Pestaña, Á, Zampieri, M., Simeoni, F., Lazzari, S., et al. (2019). Histone modifications drive aberrant Notch3 expression/activity and growth in T-ALL. Front. Oncol. 9:198. doi: 10.3389/fonc. 2019.00198

Tran, B. M., Flanagan, D. J., Ebert, G., Warner, N., Tran, H., Fifis, T., et al. (2020). The hepatitis B virus pre-core protein p22 activates Wnt signaling. Cancers (Basel) 12:1435. doi: 10.3390/cancers12061435

Trivedi, P., Slack, F. J., and Anastasiadou, E. (2018). Epstein-Barr virus: from kisses to cancer, an ingenious immune evader. Oncotarget 9, 36411-36412. doi: 10.18632/oncotarget.26381

Tukachinsky, H., Petrov, K., Watanabe, M., and Salic, A. (2016). Mechanism of inhibition of the tumor suppressor Patched by Sonic Hedgehog. Proc. Natl. Acad. Sci. U.S.A. 113, E5866-E5875. doi: 10.1073/pnas.1606719113

Umer, M., Qureshi, S. A., Hashmi, Z. Y., Raza, A., Ahmad, J., Rahman, M., et al. (2014). Promoter hypermethylation of Wnt pathway inhibitors in hepatitis C virus - induced multistep hepatocarcinogenesis. Virol. J. 11:117. doi: 10.1186/ 1743-422x-11-117

van der Meide, W. F., Snellenberg, S., Meijer, C. J., Baalbergen, A., Helmerhorst, T. J., van der Sluis, W. B., et al. (2011). Promoter methylation analysis of $\mathrm{WNT} / \beta$-catenin signaling pathway regulators to detect adenocarcinoma or its precursor lesion of the cervix. Gynecol. Oncol. 123, 116-122. doi: 10.1016/j. ygyno.2011.06.015

Vishnoi, K., Mahata, S., Tyagi, A., Pandey, A., Verma, G., Jadli, M., et al. (2016). Cross-talk between human papillomavirus oncoproteins and hedgehog 
signaling synergistically promotes stemness in cervical cancer cells. Sci. Rep. 6:34377. doi: $10.1038 /$ srep34377

Wang, H., Zang, C., Liu, X. S., and Aster, J. C. (2015). The role of Notch receptors in transcriptional regulation. J. Cell. Physiol. 230, 982-988. doi: 10.1002/jcp.24872

White, M. K., Pagano, J. S., and Khalili, K. (2014). Viruses and human cancers: a long road of discovery of molecular paradigms. Clin. Microbiol. Rev. 27, 463-481. doi: 10.1128/cmr.00124-13

Xiang, J., Rauch, D. A., Huey, D. D., Panfil, A. R., Cheng, X., Esser, A. K., et al. (2019). HTLV-1 viral oncogene HBZ drives bone destruction in adult T cell leukemia. JCI Insight 4:e128713. doi: 10.1172/jci.insight.128713

Yamamizu, K., Matsunaga, T., Uosaki, H., Fukushima, H., Katayama, S., HiraokaKanie, M., et al. (2010). Convergence of Notch and beta-catenin signaling induces arterial fate in vascular progenitors. J. Cell Biol. 189, 325-338. doi: 10.1083/jcb.200904114

Yang, L., Ye, S., Zhao, X., Ji, L., Zhang, Y., Zhou, P., et al. (2018). Molecular characterization of HBV DNA integration in patients with hepatitis and hepatocellular carcinoma. J. Cancer 9, 3225-3235. doi: 10.7150/jca.26052

Yang, P., Li, Q. J., Feng, Y., Zhang, Y., Markowitz, G. J., Ning, S., et al. (2012). TGF$\beta$-miR-34a-CCL22 signaling-induced Treg cell recruitment promotes venous metastases of HBV-positive hepatocellular carcinoma. Cancer Cell 22, 291-303. doi: 10.1016/j.ccr.2012.07.023

Yang, S., Wu, Y., Wang, S., Xu, P., Deng, Y., Wang, M., et al. (2020). HPV-related methylation-based reclassification and risk stratification of cervical cancer. Mol. Oncol. 14, 2124-2141. doi: 10.1002/1878-0261.12709

Yavropoulou, M. P., and Yovos, J. G. (2014). The role of Notch signaling in bone development and disease. Hormones (Athens) 13, 24-37. doi: 10.1007/ bf03401318

Yu, D., Han, G. H., Zhao, X., Liu, X., Xue, K., Wang, D., et al. (2020). MicroRNA$129-5 p$ suppresses nasopharyngeal carcinoma lymphangiogenesis and lymph node metastasis by targeting ZIC2. Cell Oncol. (Dordr.) 43, 249-261. doi: 10. 1007/s13402-019-00485-5

Zema, S., Pelullo, M., Nardozza, F., Felli, M. P., Screpanti, I., and Bellavia, D. (2020). A dynamic role of mastermind-like 1: a journey through the main (Path)ways between development and cancer. Front. Cell Dev. Biol. 8:613557. doi: $10.3389 /$ fcell.2020.613557
Zhang, Y., Fan, J., Fan, Y., Li, L., He, X., Xiang, Q., et al. (2018). The new 6 q27 tumor suppressor DACT2, frequently silenced by CpG methylation, sensitizes nasopharyngeal cancer cells to paclitaxel and 5-FU toxicity via $\beta$ catenin/Cdc25c signaling and G2/M arrest. Clin. Epigenetics 10:26. doi: 10.1186/ s13148-018-0459-2

Zhang, Y., Wei, W., Cheng, N., Wang, K., Li, B., Jiang, X., et al. (2012). Hepatitis C virus-induced up-regulation of microRNA-155 promotes hepatocarcinogenesis by activating Wnt signaling. Hepatology 56, 1631-1640. doi: 10.1002/hep.25 849

Zhou, S. J., Deng, Y. L., Liang, H. F., Jaoude, J. C., and Liu, F. Y. (2017). Hepatitis B virus $\mathrm{X}$ protein promotes CREB-mediated activation of miR-3188 and Notch signaling in hepatocellular carcinoma. Cell Death Differ. 24, 1577-1587. doi: 10.1038/cdd.2017.87

Zimber-Strobl, U., and Strobl, L. J. (2001). EBNA2 and Notch signalling in EpsteinBarr virus mediated immortalization of B lymphocytes. Semin. Cancer Biol. 11, 423-434. doi: 10.1006/scbi.2001.0409

Conflict of Interest: The authors declare that the research was conducted in the absence of any commercial or financial relationships that could be construed as a potential conflict of interest.

Publisher's Note: All claims expressed in this article are solely those of the authors and do not necessarily represent those of their affiliated organizations, or those of the publisher, the editors and the reviewers. Any product that may be evaluated in this article, or claim that may be made by its manufacturer, is not guaranteed or endorsed by the publisher.

Copyright (c) 2021 Trivedi, Patel, Bellavia, Messina, Palermo, Ceccarelli, Marchese, Anastasiadou, Minter and Felli. This is an open-access article distributed under the terms of the Creative Commons Attribution License (CC BY). The use, distribution or reproduction in other forums is permitted, provided the original author(s) and the copyright owner(s) are credited and that the original publication in this journal is cited, in accordance with accepted academic practice. No use, distribution or reproduction is permitted which does not comply with these terms. 